\title{
On One Approach to Investigation of Mechanical Systems
}

\author{
Valentin D. IRTEGOV and Tatyana N. TITORENKO
}

Institute for Systems Dynamics and Control Theory, SB RAS, Irkutsk, Russia

E-mail: irteg@icc.ru

Received November 18, 2005, in final form April 11, 2006; Published online May 08, 2006

Original article is available at http://www.emis.de/journals/SIGMA/2006/Paper049/

\begin{abstract}
The paper presents some results of qualitative analysis of Kirchhoff's differential equations describing motion of a rigid body in ideal fluid in Sokolov's case. The research methods are based on Lyapunov's classical results. Methods of computer algebra implemented in the computer algebra system (CAS) "Mathematica" were also used. Combination of these methods allowed us to obtain rather detailed information on qualitative properties for some classes of solutions of the equations.
\end{abstract}

Key words: rigid body mechanics; completely integrable systems; qualitative analysis; invariant manifolds; stability; bifurcations; computer algebra

2000 Mathematics Subject Classification: 37N05; 34D20; 68W30

\section{Introduction}

Let us consider the problem which may be of interest both for the development of the method of investigation employed and for numerous applications. In the problem proposed for consideration the equations of Euler-Poisson's type are the model of the object under investigation.

Euler-Poisson's differential equations describing the motion of a rigid body with one fixed point and their numerous generalizations represent one of successful mathematical models that is widely used in investigations of diverse physical phenomena and processes.

For example, the following Euler's equation for an abstract model of an infinite-dimensional dissipative top

$$
\frac{d}{d t} A \psi+\varepsilon B \psi+[\psi, A \psi]=\varepsilon f
$$

where $[\psi, A \psi]$ is Poisson's bracket, may be used to describe nonsteady-state flat-parallel flow of a viscous incompressible fluid in a channel with solid walls. The equation describing the fluid motion writes:

$$
-\frac{\partial}{\partial t} \Delta \psi+\varepsilon \Delta \Delta \psi-\frac{\partial \psi}{\partial y} \frac{\partial \Delta \psi}{\partial x}+\frac{\partial \psi}{\partial x} \frac{\partial \Delta \psi}{\partial y}=\varepsilon \cos y
$$

where $\psi(t, x, y)$ is a function of current, $\Delta=\partial^{2} / \partial x^{2}+\partial^{2} / \partial y^{2}$ is the Laplace operator.

Under the following boundary conditions
1) $0<x<\frac{2 \pi}{\alpha}, \quad 0<y<2 \pi, \quad \alpha>0$,
2) $\psi\left(t, x+\frac{2 \pi}{\alpha}, y\right)=\psi(t, x, y)$,
3) $\left.\psi\right|_{y=0}=\left.\frac{\partial \psi}{\partial y}\right|_{y=0}=\left.\psi\right|_{y=2 \pi}=\left.\frac{\partial \psi}{\partial y}\right|_{y=2 \pi}$ 
(condition 3 indicates to the zero fluid flow rate) and the following correlation between the operators

$$
A \equiv-\Delta, \quad B \equiv \Delta \Delta, \quad[\psi, \varphi] \equiv \frac{\partial \psi}{\partial y} \frac{\partial \varphi}{\partial x}-\frac{\partial \psi}{\partial x} \frac{\partial \varphi}{\partial y},
$$

equation (2) is similar to (1).

Such analogies allow one to conduct, for example, analysis of stability in problems of above type by classical methods of rigid body dynamics, and to suggest clear interpretation of results obtained [10].

The present paper represents some results of qualitative analysis of the differential equations describing the motion of a rigid body in ideal incompressible fluid. If the following conditions are satisfied here, i.e. the fluid possesses a single-valued potential of rates and rests at infinity, then the body motion equations (6 ODEs) separate from the partial differential equations which describe the motion of fluid. In this case, the motion equations of the body coincide in their form with the corresponding Euler-Poisson equations and are called Kirchhoff's equations [4, 7]:

$$
\dot{M}=M \times \frac{\partial H}{\partial M}+\gamma \times \frac{\partial H}{\partial \gamma}, \quad \dot{\gamma}=\gamma \times \frac{\partial H}{\partial M},
$$

where $M=\left(M_{1}, M_{2}, M_{3}\right), \gamma=\left(\gamma_{1}, \gamma_{2}, \gamma_{3}\right)$ are vectors of "impulse moment" and "impulsive force", respectively.

The total kinetic energy for the body and the fluid writes:

$$
2 T=2 H=(A M, M)+2(B M, \gamma)+(C \gamma, \gamma) .
$$

Here $A, B, C$ are constant matrices. The latter are inertial characteristics of the body and the fluid. By a special choice of the origin and the direction of axes in the body it is possible to make the matrix $A$ diagonal, and the matrix $C$ symmetric, respectively.

In problems of qualitative analysis of Euler's equations, it is possible to obtain the most complete results when the equations have many first integrals, for example, the equations are completely integrable. In this case, the phase space of system of the equations has a simple structure.

We consider Kirchhoff's differential equations in Sokolov's case [11]. The equations in this case represent a completely integrable system. These write:

$$
\begin{aligned}
& \dot{M}_{1}=M_{2} M_{3}+\alpha\left(\gamma_{2} M_{1}+\gamma_{1} M_{2}\right)+2 \beta\left(\gamma_{2} M_{2}-\gamma_{3} M_{3}\right)-4 \gamma_{2} \gamma_{3}\left(2 \alpha^{2}+\beta^{2}\right)+4 \alpha \beta \gamma_{1} \gamma_{3}, \\
& \dot{M}_{2}=4 \gamma_{1} \gamma_{3}\left(\alpha^{2}+2 \beta^{2}\right)-\beta\left(\gamma_{2} M_{1}+\gamma_{1} M_{2}\right)-M_{1} M_{3}-2 \alpha\left(\gamma_{1} M_{1}-\gamma_{3} M_{3}\right)-4 \alpha \beta \gamma_{2} \gamma_{3}, \\
& \dot{M}_{3}=4 \gamma_{1} \gamma_{2}\left(\alpha^{2}-\beta^{2}\right)+\beta\left(\gamma_{3} M_{1}+\gamma_{1} M_{3}\right)-\alpha\left(\gamma_{3} M_{2}+\gamma_{2} M_{3}\right)-4 \alpha \beta\left(\gamma_{1}^{2}-\gamma_{2}^{2}\right), \\
& \dot{\gamma}_{1}=\gamma_{2}\left(2 M_{3}+\alpha \gamma_{1}\right)+\beta\left(\gamma_{2}^{2}-\gamma_{3}^{2}\right)-\gamma_{3} M_{2}, \\
& \dot{\gamma}_{2}=-\gamma_{1}\left(2 M_{3}+\beta \gamma_{2}\right)-\alpha\left(\gamma_{1}^{2}-\gamma_{3}^{2}\right)+\gamma_{3} M_{1}, \\
& \dot{\gamma}_{3}=\gamma_{1}\left(M_{2}+\beta \gamma_{3}\right)-\gamma_{2}\left(M_{1}+\alpha \gamma_{3}\right) .
\end{aligned}
$$

Here $\alpha, \beta$ are arbitrary constants.

The system (3) has the following 4 algebraic first integrals:

$$
\begin{aligned}
2 H= & M_{1}^{2}+M_{2}^{2}+2 M_{3}^{2}+2 \alpha\left(\gamma_{3} M_{1}+\gamma_{1} M_{3}\right)+2 \beta\left(\gamma_{3} M_{2}+\gamma_{2} M_{3}\right)+4\left(\beta \gamma_{1}-\alpha \gamma_{2}\right)^{2} \\
& -4 \gamma_{3}^{2}\left(\alpha^{2}+\beta^{2}\right)=2 h, \\
V_{1}= & \gamma_{1} M_{1}+\gamma_{2} M_{2}+\gamma_{3} M_{3}=c_{1}, \quad V_{2}=\gamma_{1}^{2}+\gamma_{2}^{2}+\gamma_{3}^{2}=c_{2}, \\
V_{3}= & \left\{3\left(\beta \gamma_{1}-\alpha \gamma_{2}\right)\left(\beta M_{1}-\alpha M_{2}\right)+\left(2 \alpha \gamma_{1}+2 \beta \gamma_{2}+M_{3}\right)\left(\left(\alpha^{2}+\beta^{2}\right) \gamma_{3}+\alpha M_{1}+\beta M_{2}\right)\right\}^{2} \\
& +\left(M_{3}-\alpha \gamma_{1}-\beta \gamma_{2}\right)^{2}\left\{\left(\beta M_{1}-\alpha M_{2}\right)^{2}+\left(\alpha^{2}+\beta^{2}\right)\left(2 \alpha \gamma_{1}+2 \beta \gamma_{2}+M_{3}\right)^{2}\right\}=c_{3} .
\end{aligned}
$$


A lot of works were devoted to investigation of the Kirchhoff's equations. A substantial part of these works is related to the problems of integrability.

Another part of such works was devoted to the problems of investigation of stability for permanent motions (in particular, helical motions) of a rigid body in ideal fluid. The first results in this direction go back to Lyapunov [8]. This investigation was further developed, and some results can be found, for example, in $[3,5,6,13]$.

The objective of the present work is to conduct qualitative analysis of solutions of equations (3). We investigate a class of solutions of the equations, on which the elements of algebra of the problem's first integrals assume stationary values. Such solutions will be called stationary (see Appendix A for details). In particular, we found families of stationary solutions and families of invariant manifolds of steady motions (IMSMs) for the system of equations under scrutiny. We obtained conditions of stability and instability for several families of stationary solutions and families of IMSMs; parametric analysis of some of these conditions was conducted. Besides, some problems of bifurcations for both the families of stationary solutions and the families of IMSMs branching from these solutions (in particular, a trivial solution) were considered. Furthermore, the character of stability for branching manifolds was taken into account.

The methods of investigation are based on classical Lyapunov's results [8, 9], in particular, on his 2nd method. Methods of computer algebra implemented in CAS "Mathematica" were also used. A combination of these methods enabled to obtain results quite interesting from our viewpoint.

\section{Obtaining stationary solutions}

Let us consider the problem of finding stationary solutions and invariant manifolds of steady motions for the system (3).

Analysis of equations (3) in terms of initial variables $M_{i}, \gamma_{i}, i=1,2,3$, is rather bulky, and hence difficult. Therefore, in the papers devoted to the analysis of above equations, different linear transformations of variables are applied allowing one to reduce the equations and the integrals to a more compact form. In the present paper we use the following linear (not degenerate) transformation of the variables from [1]:

$$
\begin{aligned}
& M_{1}=s_{1}-\frac{1}{3} \tilde{\alpha} r_{3}, \quad M_{2}=s_{2}-\frac{1}{3} \tilde{\beta} r_{3}, \quad M_{3}=s_{3}+\frac{1}{3} \tilde{\alpha} r_{1}+\frac{1}{3} \tilde{\beta} r_{2}, \\
& \gamma_{i}=r_{i}, \quad \alpha=\frac{1}{3} \tilde{\alpha}, \quad \beta=\frac{1}{3} \tilde{\beta}, \quad i=1,2,3 .
\end{aligned}
$$

The latter enabled us to find out stationary solutions and IMSMs for the system (3) and to perform their analysis without going beyond standard algorithms.

On account of the linear transformation of the variables (5), the equations of motion (3) for $\beta=0$ will take the form:

$$
\begin{array}{ll}
\dot{r}_{1}=\left(\alpha r_{1}+2 s_{3}\right) r_{2}-r_{3} s_{2}, & \dot{s}_{1}=\left(\alpha r_{1}+s_{3}\right) s_{2}-\alpha^{2} r_{2} r_{3}, \\
\dot{r}_{2}=r_{3} s_{1}-r_{1}\left(\alpha r_{1}+2 s_{3}\right), & \dot{s}_{2}=\left(\alpha r_{3}-s_{1}\right)\left(\alpha r_{1}+s_{3}\right), \\
\dot{r}_{3}=r_{1} s_{2}-r_{2} s_{1}, & \dot{s}_{3}=-\alpha r_{2} s_{3},
\end{array}
$$

and the corresponding first integrals write:

$$
\begin{aligned}
& 2 H=\left(s_{1}^{2}+s_{2}^{2}+2 s_{3}^{2}\right)+2 \alpha r_{1} s_{3}-\alpha^{2} r_{3}^{2}=2 h, \\
& V_{1}=s_{1} r_{1}+s_{2} r_{2}+s_{3} r_{3}=c_{1}, \quad V_{2}=r_{1}^{2}+r_{2}^{2}+r_{3}^{2}=c_{2}, \\
& 2 V_{3}=\left(\alpha r_{1} s_{1}+\alpha r_{2} s_{2}+s_{1} s_{3}\right)^{2}+s_{3}^{2}\left(s_{2}^{2}+\left(\alpha r_{1}+s_{3}\right)^{2}\right)=2 c_{3} .
\end{aligned}
$$


We shall now consider the problem of finding stationary solutions and IMSMs for the system (6). We shall apply Routh-Lyapunov's method [12] (see also Appendix B) for solving it. This will allow one to perform a substantial part of computational work with the use of computer algebra systems.

In accordance with the method of Routh-Lyapunov, the functions $K$ are constructed on the basis of the problem's first integrals. We shall construct here linear combinations of these integrals only (the combinations may be nonlinear ones):

$$
K=\lambda_{0} H-\lambda_{1} V_{1}-\lambda_{2} V_{2}-\lambda_{3} V_{3}, \quad \lambda_{i}=\text { const. }
$$

The integral $K$ represents a family of first integrals, which is parametrized by the values $\lambda_{0}, \lambda_{1}$, $\lambda_{2}, \lambda_{3}$. We enter an excessive number of parameters into $K$ that allows us to obtain "incomplete" combinations of integrals by equating some part of the parameters in $K$ to zero. It is worth to note that different elements of algebra of first integrals will, generally speaking, correspond to various stationary solutions and manifolds.

Next, we write down stationary conditions for $K$ with respect to all the variables $s_{1}, s_{2}, s_{3}$, $r_{1}, r_{2}, r_{3}:$

$$
\begin{aligned}
\frac{\partial K}{\partial s_{1}}= & \lambda_{0} s_{1}-\lambda_{1} r_{1}-\alpha^{2} \lambda_{3} r_{1}^{2} s_{1}-\alpha^{2} \lambda_{3} r_{1} r_{2} s_{2}-2 \alpha \lambda_{3} r_{1} s_{1} s_{3}-\alpha \lambda_{3} r_{2} s_{2} s_{3}-\lambda_{3} s_{1} s_{3}^{2}=0 \\
\frac{\partial K}{\partial s_{2}}= & \lambda_{0} s_{2}-\lambda_{1} r_{2}-\alpha^{2} \lambda_{3} r_{1} r_{2} s_{1}-\alpha^{2} \lambda_{3} r_{2}^{2} s_{2}-\alpha \lambda_{3} r_{2} s_{1} s_{3}-\lambda_{3} s_{2} s_{3}^{2}=0 \\
\frac{\partial K}{\partial s_{3}}= & \alpha \lambda_{0} r_{1}-\lambda_{1} r_{3}-\alpha \lambda_{3} r_{1} s_{1}^{2}-\alpha \lambda_{3} r_{2} s_{1} s_{2}+2 \lambda_{0} s_{3}-\alpha^{2} \lambda_{3} r_{1}^{2} s_{3}-\lambda_{3} s_{1}^{2} s_{3}-\lambda_{3} s_{2}^{2} s_{3} \\
& -3 \alpha \lambda_{3} r_{1} s_{3}^{2}-2 \lambda_{3} s_{3}^{3}=0 \\
\frac{\partial K}{\partial r_{1}}= & \alpha \lambda_{0} s_{3}-2 \lambda_{2} r_{1}-\lambda_{1} s_{1}-\alpha^{2} \lambda_{3} r_{1} s_{1}^{2}-\alpha^{2} \lambda_{3} r_{2} s_{1} s_{2}-\alpha \lambda_{3} s_{1}^{2} s_{3}-\alpha^{2} \lambda_{3} r_{1} s_{3}^{2}-\alpha \lambda_{3} s_{3}^{3}=0 \\
\frac{\partial K}{\partial r_{2}}= & 2 \lambda_{2} r_{2}+\lambda_{1} s_{2}+\alpha^{2} \lambda_{3} r_{1} s_{1} s_{2}+\alpha^{2} \lambda_{3} r_{2} s_{2}^{2}+\alpha \lambda_{3} s_{1} s_{2} s_{3}=0 \\
\frac{\partial K}{\partial r_{3}}= & \left(\alpha^{2} \lambda_{0}+2 \lambda_{2}\right) r_{3}+\lambda_{1} s_{3}=0
\end{aligned}
$$

Solutions of system (9) define stationary solutions and IMSMs for the system (6) (see Appendices A, B). In the general case these solutions may contain parameters $\lambda_{i}$ that appear in the family of integrals $K(8)$, and may hence represent a family of stationary solutions and IMSMs. Hence, to solve the problem formulated (obtaining stationary solutions and IMSMs for the system (6) corresponding to the family of first integrals $K$ ) it is necessary to solve the system of 6 algebraic equations containing four parameters $\lambda_{0}, \lambda_{1}, \lambda_{2}, \lambda_{3}$. The number of variables is 6 . The system will have a closed form if any three first integrals (7) are added to it. In particular, this means that the parameters $\lambda_{i}$ may be obtained as some functions of the constants of first integrals.

All the equations of system (9) are nonlinear, except for the last one that allows us to slightly simplify the problem. After removing the variable $r_{3}$ from the remaining equations (9) with the use of the last one, we obtain the following system of 5 nonlinear algebraic equations containing 5 variables:

$$
\begin{aligned}
& \lambda_{0} s_{1}-\lambda_{1} r_{1}-\alpha^{2} \lambda_{3} r_{1}^{2} s_{1}-\alpha^{2} \lambda_{3} r_{1} r_{2} s_{2}-2 \alpha \lambda_{3} r_{1} s_{1} s_{3}-\alpha \lambda_{3} r_{2} s_{2} s_{3}-\lambda_{3} s_{1} s_{3}^{2}=0, \\
& \lambda_{0} s_{2}-\lambda_{1} r_{2}-\alpha^{2} \lambda_{3} r_{1} r_{2} s_{1}-\alpha^{2} \lambda_{3} r_{2}^{2} s_{2}-\alpha \lambda_{3} r_{2} s_{1} s_{3}-\lambda_{3} s_{2} s_{3}^{2}=0 \\
& \alpha \lambda_{0} r_{1}-\frac{\lambda_{1}^{2}}{\alpha^{2} \lambda_{0}+2 \lambda_{2}} s_{3}-\alpha \lambda_{3} r_{1} s_{1}^{2}-\alpha \lambda_{3} r_{2} s_{1} s_{2}+2 \lambda_{0} s_{3}-\alpha^{2} \lambda_{3} r_{1}^{2} s_{3}-\lambda_{3} s_{1}^{2} s_{3}
\end{aligned}
$$




$$
\begin{aligned}
& \quad-\lambda_{3} s_{2}^{2} s_{3}-3 \alpha \lambda_{3} r_{1} s_{3}^{2}-2 \lambda_{3} s_{3}^{3}=0, \\
& \alpha \lambda_{0} s_{3}-2 \lambda_{2} r_{1}-\lambda_{1} s_{1}-\alpha^{2} \lambda_{3} r_{1} s_{1}^{2}-\alpha^{2} \lambda_{3} r_{2} s_{1} s_{2}-\alpha \lambda_{3} s_{1}^{2} s_{3}-\alpha^{2} \lambda_{3} r_{1} s_{3}^{2}-\alpha \lambda_{3} s_{3}^{3}=0, \\
& 2 \lambda_{2} r_{2}+\lambda_{1} s_{2}+\alpha^{2} \lambda_{3} r_{1} s_{1} s_{2}+\alpha^{2} \lambda_{3} r_{2} s_{2}^{2}+\alpha \lambda_{3} s_{1} s_{2} s_{3}=0 .
\end{aligned}
$$

The maximum degree of the equations belonging to the system is 3 .

To the end of obtaining solutions of system (10), we apply the Gröbner bases (GB) method [2] traditionally used in computer algebra for solving similar systems. Software implementation of the method can be found in many CAS. Application of only standard tools of CAS "Mathematica" for computing the GB allowed us to construct the Gröbner basis for the system (10) under the following lexicographic ordering of the variables: $r_{1}>r_{2}>s_{2}>s_{1}>s_{3}$. The timing for construction of the basis, as measured on a $1100 \mathrm{MHz}$ Pentium with $256 \mathrm{MB}$ RAM running under Windows XP, is 2.71 seconds. Below one can find a structure of Gröbner's basis constructed under the indicated ranging of the variables for the given system of equations.

$$
\begin{aligned}
& s_{2} s_{3} f_{1}\left(s_{1}, s_{3}\right)=0, \quad\left(\left(\alpha^{2} \lambda_{0}+2 \lambda_{2}\right) s_{1}+\alpha \lambda_{1} s_{3}\right) f_{2}\left(s_{1}, s_{3}\right)=0, \quad s_{2} f_{3}\left(s_{1}, s_{2}, s_{3}\right)=0 \\
& s_{3} f_{4}\left(s_{1}, s_{3}\right) f_{5}\left(s_{3}\right)=0, \quad f_{6}\left(r_{2}, s_{1}, s_{2}, s_{3}\right)=0, \quad s_{3} f_{7}\left(s_{1}, s_{2}, s_{3}\right)=0 \\
& f_{8}\left(s_{1}, s_{2}, s_{3}\right)=0, \quad f_{9}\left(r_{1}, s_{1}, s_{3}\right)=0 .
\end{aligned}
$$

Here $f_{i}, i=1, \ldots, 9$, are polynomials of the variables $s_{1}, s_{2}, s_{3}, r_{1}, r_{2}$. The maximum degree of the polynomials is 7 . These are too bulky, and so are omitted here. The system (11) is given in complete form in Appendix C.

As is obvious from (11), the basis constructed can easily be factorized that allows to decompose it into several subsystems, which may be analyzed separately. Up to 12 subsystems were identified. For each of the subsystems we constructed a Gröbner basis under lexicographic ordering of the variables. The latter enabled us to conduct some qualitative analysis of the set of solutions of each subsystem (with respect to the compatibility, finiteness or infiniteness of the set of the subsystems' solutions, etc.) and hence to obtain information about the whole set of system's (11) solutions (respectively, (10) and (9)) and find out some groups of solutions.

Finally, we conclude that the system (9) has an infinite set of solutions (the variable $s_{3}$ is free). The following groups of solutions (besides the trivial solution) were found out: 4 families of IMSMs and 8 families of stationary solutions. Some of the solutions obtained can be found below. The solutions are given in the form representing the result of computing.

1. The families of invariant manifolds of steady motions:

$$
\begin{aligned}
& \left\{\left\{s_{1}=-\frac{\sqrt{2 \lambda_{2}}\left(\alpha^{2} \lambda_{0}+2 \lambda_{2}\right) \sqrt{\lambda_{3} s_{3}^{2}-\lambda_{0}}+\lambda_{1}\left(2 \lambda_{2}+\alpha^{2} \lambda_{3} s_{3}^{2}\right)}{\alpha\left(\alpha^{2} \lambda_{0}+2 \lambda_{2}\right) \lambda_{3} s_{3}},\right.\right. \\
& s_{2}=\mp \frac{\sqrt{2 \lambda_{2} z_{1}}}{\alpha\left(\alpha^{2} \lambda_{0}+2 \lambda_{2}\right) \lambda_{3} s_{3}}, \quad r_{1}=-\frac{\sqrt{\lambda_{3} s_{3}^{2}-\lambda_{0}}\left(\left(\alpha^{2} \lambda_{0}+2 \lambda_{2}\right) \sqrt{\lambda_{3} s_{3}^{2}-\lambda_{0}}+\sqrt{2 \lambda_{2}} \lambda_{1}\right)}{\alpha\left(\alpha^{2} \lambda_{0}+2 \lambda_{2}\right) \lambda_{3} s_{3}}, \\
& \left.r_{2}=\mp \frac{\sqrt{\left(\lambda_{3} s_{3}^{2}-\lambda_{0}\right) z_{1}}}{\alpha\left(\alpha^{2} \lambda_{0}+2 \lambda_{2}\right) \lambda_{3} s_{3}}, \quad r_{3}=-\frac{\lambda_{1} s_{3}}{\alpha^{2} \lambda_{0}+2 \lambda_{2}}\right\}, \\
& \left\{s_{1}=\frac{\sqrt{2 \lambda_{2}}\left(\alpha^{2} \lambda_{0}+2 \lambda_{2}\right) \sqrt{\lambda_{3} s_{3}^{2}-\lambda_{0}}-\lambda_{1}\left(2 \lambda_{2}+\alpha^{2} \lambda_{3} s_{3}^{2}\right)}{\alpha\left(\alpha^{2} \lambda_{0}+2 \lambda_{2}\right) \lambda_{3} s_{3}}, \quad s_{2}=\mp \frac{\sqrt{2 \lambda_{2} z_{2}}}{\alpha\left(\alpha^{2} \lambda_{0}+2 \lambda_{2}\right) \lambda_{3} s_{3}},\right. \\
& r_{1}=-\frac{\sqrt{\lambda_{3} s_{3}^{2}-\lambda_{0}}\left(\left(\alpha^{2} \lambda_{0}+2 \lambda_{2}\right) \sqrt{\lambda_{3} s_{3}^{2}-\lambda_{0}}-\sqrt{2 \lambda_{2}} \lambda_{1}\right)}{\alpha\left(\alpha^{2} \lambda_{0}+2 \lambda_{2}\right) \lambda_{3} s_{3}}, \quad r_{2}= \pm \frac{\sqrt{\left(\lambda_{3} s_{3}^{2}-\lambda_{0}\right) z_{2}}}{\alpha\left(\alpha^{2} \lambda_{0}+2 \lambda_{2}\right) \lambda_{3} s_{3}}
\end{aligned}
$$


For brevity, we introduced the following denotations:

$$
\begin{aligned}
z_{1}= & \alpha^{4} \lambda_{0}^{3}+4 \alpha^{2} \lambda_{0}^{2} \lambda_{2}-2 \lambda_{1}^{2} \lambda_{2}+4 \lambda_{0} \lambda_{2}^{2}-\left(\alpha^{4} \lambda_{0}^{2}+4 \lambda_{2}^{2}+\alpha^{2}\left(\lambda_{1}^{2}+4 \lambda_{0} \lambda_{2}\right)\right) \lambda_{3} s_{3}^{2} \\
& -2 \sqrt{2 \lambda_{2}} \lambda_{1}\left(\alpha^{2} \lambda_{0}+2 \lambda_{2}\right) \sqrt{\lambda_{3} s_{3}^{2}-\lambda_{0}}, \\
z_{2}= & \alpha^{4} \lambda_{0}^{3}+4 \alpha^{2} \lambda_{0}^{2} \lambda_{2}-2 \lambda_{1}^{2} \lambda_{2}+4 \lambda_{0} \lambda_{2}^{2}-\left(\alpha^{4} \lambda_{0}^{2}+4 \lambda_{2}^{2}+\alpha^{2}\left(\lambda_{1}^{2}+4 \lambda_{0} \lambda_{2}\right)\right) \lambda_{3} s_{3}^{2} \\
& +2 \sqrt{2 \lambda_{2}} \lambda_{1}\left(\alpha^{2} \lambda_{0}+2 \lambda_{2}\right) \sqrt{\lambda_{3} s_{3}^{2}-\lambda_{0}} .
\end{aligned}
$$

2. The families of stationary solutions:

$$
\begin{aligned}
& \left\{\left\{r_{1}=0, \quad r_{2}= \pm \frac{\sqrt{\lambda_{1} \sqrt{-2 \lambda_{0} \lambda_{2}}+2 \lambda_{0} \lambda_{2}}}{\sqrt{2} \alpha \sqrt{\lambda_{2} \lambda_{3}}}, \quad r_{3}=0, \quad s_{1}=0,\right.\right. \\
& \left.s_{2}=\mp \frac{\sqrt{\lambda_{1} \sqrt{-2 \lambda_{2}}+2 \lambda_{2} \sqrt{\lambda_{0}}}}{\alpha \sqrt{-\sqrt{\lambda_{0}} \lambda_{3}}}, \quad s_{3}=0\right\}, \\
& \left\{r_{1}= \pm \frac{\sqrt{-z_{3}\left(2 \lambda_{2}+\sqrt{z_{3}}\right)}\left(\alpha^{2} \lambda_{0}+2 \lambda_{2}+\sqrt{z_{3}}\right)}{\alpha^{2} z_{3} \sqrt{\lambda_{3}}}, \quad r_{2}=0,\right. \\
& r_{3}= \pm \frac{\lambda_{1} \sqrt{-z_{3}\left(2 \lambda_{2}+\sqrt{z_{3}}\right)}}{\alpha z_{3} \sqrt{\lambda_{3}}}, \quad s_{1}= \pm \frac{\left.\lambda_{1} \sqrt{-z_{3}\left(2 \lambda_{2}+\sqrt{z_{3}}\right.}\right)}{z_{3} \sqrt{\lambda_{3}}}, \quad s_{2}=0, \\
& \left.\left.s_{3}=\mp \frac{\left(\alpha^{2} \lambda_{0}+2 \lambda_{2}\right) \sqrt{-z_{3}\left(2 \lambda_{2}+\sqrt{z_{3}}\right)}}{\alpha z_{3} \sqrt{\lambda_{3}}}\right\}\right\} .
\end{aligned}
$$

Here $z_{3}=\alpha^{4} \lambda_{0}^{2}+4 \lambda_{2}^{2}+\alpha^{2}\left(\lambda_{1}^{2}+4 \lambda_{0} \lambda_{2}\right)$.

Tools of computer algebra allow us to write rather easily the obtained solutions in terms of variables of any other "good" coordinate system, for example, in terms of the initial variables $M_{i}, \gamma_{i}$. The solutions remain stationary also in terms of these variables.

The families of stationary solutions (13) are given in terms of the variables $M_{1}, M_{2}, M_{3}, \gamma_{1}$, $\gamma_{2}, \gamma_{3}$ :

$$
\begin{aligned}
& \left\{\left\{\gamma_{1}=0, \quad \gamma_{2}= \pm \frac{\sqrt{\lambda_{1} \sqrt{-2 \lambda_{0} \lambda_{2}}+2 \lambda_{0} \lambda_{2}}}{3 \sqrt{2} \alpha \sqrt{\lambda_{2} \lambda_{3}}}, \quad \gamma_{3}=0, \quad M_{1}=0,\right.\right. \\
& \left.M_{2}=\mp \frac{\sqrt{\lambda_{1} \sqrt{-2 \lambda_{2}}+2 \sqrt{\lambda_{0}} \lambda_{2}}}{3 \alpha \sqrt{-\sqrt{\lambda_{0}} \lambda_{3}}}, \quad M_{3}=0\right\}, \\
& \left\{\gamma_{1}= \pm \frac{\sqrt{-p_{1}\left(2 \lambda_{2}+\sqrt{p_{1}}\right)}\left(9 \alpha^{2} \lambda_{0}+2 \lambda_{2}+\sqrt{p_{1}}\right)}{9 \alpha^{2} p_{1} \sqrt{\lambda_{3}}}, \quad \gamma_{2}=0,\right. \\
& \gamma_{3}= \pm \frac{\lambda_{1} \sqrt{-p_{1}\left(2 \lambda_{2}+\sqrt{p_{1}}\right)}}{3 \alpha p_{1} \sqrt{\lambda_{3}}}, \quad M_{1}= \pm \frac{2 \lambda_{1} \sqrt{-p_{1}\left(2 \lambda_{2}+\sqrt{p_{1}}\right)}}{3 p_{1} \sqrt{\lambda_{3}}}, \quad M_{2}=0, \\
& \left.\left.M_{3}=\mp \frac{\left(18 \alpha^{2} \lambda_{0}+4 \lambda_{2}-\sqrt{p_{1}}\right) \sqrt{-p_{1}\left(2 \lambda_{2}+\sqrt{p_{1}}\right)}}{9 \alpha p_{1} \sqrt{\lambda_{3}}}\right\}\right\} .
\end{aligned}
$$

Here $p_{1}=81 \alpha^{4} \lambda_{0}^{2}+4 \lambda_{2}^{2}+9 \alpha^{2}\left(\lambda_{1}^{2}+4 \lambda_{0} \lambda_{2}\right)$.

Analysis of IMSMs (12) showed that, after transforming these expressions to the form, which does not contain the problem's variables under radicals, we obtain one family of IMSMs. The system of equalities (12) is a representation of the latter in different maps only. The equations, which define the family of IMSMs, write:

$$
\alpha^{4} \lambda_{1}^{2} \lambda_{3}^{2} s_{3}^{4}+2 a_{11} \alpha^{3} \lambda_{1} \lambda_{3}^{2} s_{1} s_{3}^{3}+a_{11}^{2} \alpha^{2} \lambda_{3}^{2} s_{1}^{2} s_{3}^{2}-2 a_{10} \lambda_{2} \lambda_{3} s_{3}^{2}+4 a_{11} \alpha \lambda_{1} \lambda_{2} \lambda_{3} s_{1} s_{3}+2 a_{6} \lambda_{2}=0,
$$




$$
\begin{aligned}
& a_{11}^{4} \alpha^{4} \lambda_{3}^{4} s_{2}^{2} s_{3}^{4}+4 a_{11}^{2} a_{9} \alpha^{2} \lambda_{2} \lambda_{3}^{3} s_{2} s_{3}^{4}+4 a_{9}^{2} \lambda_{2}^{2} \lambda_{3}^{2} s_{3}^{4}-4 a_{11}^{2} a_{8} \alpha^{2} \lambda_{2} \lambda_{3}^{2} s_{2} s_{3}^{2} \\
& \quad-8 a_{4} \lambda_{2}^{2} \lambda_{3} s_{3}^{2}+4 a_{6}^{2} \lambda_{2}^{2}=0 \\
& a_{11}^{2} \lambda_{3}^{2} s_{3}^{4}+2 a_{11}^{2} \alpha \lambda_{3}^{2} r_{1} s_{3}^{3}+a_{11}^{2} \alpha^{2} \lambda_{3}^{2} r_{1}^{2} s_{3}^{2}-2 a_{7} \lambda_{3} s_{3}^{2}-2 a_{11}^{2} \alpha \lambda_{0} \lambda_{3} r_{1} s_{3}+a_{6} \lambda_{0}=0, \\
& a_{9}^{2} \lambda_{3}^{4} s_{3}^{8}+2 a_{11}^{2} a_{9} \alpha^{2} \lambda_{3}^{4} r_{2} s_{3}^{6}-2 a_{3} \lambda_{3}^{3} s_{3}^{6}+a_{11}^{4} \alpha^{4} \lambda_{3}^{4} r_{2}^{2} s_{3}^{4}-2 a_{11}^{2} a_{5} \alpha^{2} \lambda_{3}^{3} r_{2} s_{3}^{4}+a_{1} \lambda_{3}^{2} s_{3}^{4} \\
& \quad+2 a_{11}^{2} a_{8} \alpha^{2} \lambda_{0} \lambda_{3}^{2} r_{2} s_{3}^{2}-2 a_{2} \lambda_{0} \lambda_{3} s_{3}^{2}+a_{6}^{2} \lambda_{0}^{2}=0, \\
& a_{11} r_{3}+\lambda_{1} s_{3}=0
\end{aligned}
$$

where $a_{i}, i=1, \ldots, 11$, are polynomials of the parameters $\lambda_{0}, \lambda_{1}, \lambda_{2}, \lambda_{3}$. These are too cumbersome, and so are omitted herein.

From the geometric viewpoint, equations (14) - for each fixed collection of parameters of the family $\lambda_{0}, \lambda_{1}, \lambda_{2}, \lambda_{3}$ - describe the curves lying in $\mathbb{R}^{6}$ at the intersection of the three 4th-order hypersurfaces, the 8 th-order hypersurface and the hyperplane.

The complete analysis of the family of IMSMs (14) and of motions on it is not given in the present paper. This analysis is rather nontrivial and may be a subject matter for another paper.

\section{Investigation of stability for stationary solutions and for IMSMs}

Let us consider the problem of stability for a series of families of stationary solutions and families of IMSMs of system (6).

\subsection{Investigation of invariant manifolds}

Consider equations (9) under the condition $\lambda_{0}=\lambda_{1}=\lambda_{3}=0$. It can be readily seen that thy have the following solution $r_{1}=r_{2}=r_{3}=0$, which defines the 3 -dimensional invariant manifold of steady motions for the equations (6). The latter allow one to define the vector field on the IMSM (i.e. to reduce the initial system of equations to the IMSM):

$$
\dot{s_{1}}=s_{2} s_{3}, \quad \dot{s_{2}}=-s_{1} s_{3}, \quad \dot{s_{3}}=0 \text {. }
$$

If we consider the initial values of the variables $s_{i}, i=1,2,3$, as parameters, it is possible to assume that here we deal with the family of invariant manifolds. Hence, for $s_{3}^{0}=0$ this family adjuncts to the zero solution of the problem, i.e. it has at least one common point with the zero solution.

When using the first integral $V_{2}=r_{1}^{2}+r_{2}^{2}+r_{3}^{2}$, which assumes the minimum value on the family of IMSMs, we easily conclude on stability of the elements of the family of IMSMs (15).

The vector field on the elements of the family of IMSMs has the two first integrals:

$$
W_{1}=s_{1}^{2}+s_{2}^{2}=m_{1}, \quad W_{2}=s_{3}=m_{2}
$$

Consequently, in this case we may speak of investigation of the 2nd-level stationary solutions (see Appendix A). Finding such solutions and their analysis are trivial in the computational aspect, and we will not concentrate on them here. Let us consider a more complex case.

\subsection{Investigation of 2nd-level stationary solutions}

By constructing the Gröbner bases with respect to the problem's variables and to one or several parameters $\lambda_{0}, \lambda_{1}, \lambda_{2}, \lambda_{3}$ for the subsystems of system (11) we can also obtain solutions under 
some conditions imposed on above parameters. For example, we constructed the Gröbner basis with respect to the variables $r_{1}, r_{2}, s_{1}$ and the parameter $\lambda_{2}$ for the subsystem

$$
\begin{aligned}
& s_{2} s_{3}=0, \quad\left(\left(\alpha^{2} \lambda_{0}+2 \lambda_{2}\right) s_{1}+\alpha \lambda_{1} s_{3}\right) f_{2}\left(s_{1}, s_{3}\right)=0, \quad s_{2}=0, \quad s_{3}=0, \\
& f_{6}\left(r_{2}, s_{1}, s_{2}, s_{3}\right)=0, \quad f_{8}\left(s_{1}, s_{2}, s_{3}\right)=0, \quad f_{9}\left(r_{1}, s_{1}, s_{3}\right)=0
\end{aligned}
$$

of system (11). It enabled us to obtain solutions of the system under the following conditions imposed on the parameter $\lambda_{2}$ :

$$
\begin{aligned}
& \lambda_{2}=-\frac{2 \alpha^{2} \lambda_{0}^{2}+\lambda_{1}^{2}+\lambda_{1} \sqrt{4 \alpha^{2} \lambda_{0}^{2}+\lambda_{1}^{2}}}{4 \lambda_{0}}, \\
& \lambda_{2}=-\frac{2 \alpha^{2} \lambda_{0}^{2}+\lambda_{1}^{2}-\lambda_{1} \sqrt{4 \alpha^{2} \lambda_{0}^{2}+\lambda_{1}^{2}}}{4 \lambda_{0}} .
\end{aligned}
$$

The solutions obtained can be found in Appendix D. The solutions are given right in the form representing the result of computing.

Likewise in case of (12), after transforming the expressions of solutions obtained to the form, which does not contain the problem's variables under radicals, we found out that these solutions represent the families of IMSMs for the system (6), these solutions being written in terms of the maps for these families. Finally, we have 2 families of IMSMs.

The first family of IMSMs, which corresponds to $\lambda_{2}(17)$, can be written as:

$$
\begin{aligned}
& 2 \alpha^{4} \lambda_{0}^{2} r_{1}^{2}+\left(2 \alpha^{2} \lambda_{0}^{2}+\lambda_{1}\left(\lambda_{1}-\sqrt{4 \alpha^{2} \lambda_{0}^{2}+\lambda_{1}^{2}}\right)\right) s_{2}^{2} \\
& \quad=\left(2 \alpha^{2} \lambda_{0}^{2}+\lambda_{1}\left(\lambda_{1}-\sqrt{4 \alpha^{2} \lambda_{0}^{2}+\lambda_{1}^{2}}\right)\right) \frac{\lambda_{0}}{\lambda_{3}} \\
& s_{1}^{2}+s_{2}^{2}=\frac{\lambda_{0}}{\lambda_{3}}, \quad s_{3}=0, \quad r_{3}=0, \quad 2 \alpha^{2} \lambda_{0} r_{2}+\left(\lambda_{1}-\sqrt{4 \alpha^{2} \lambda_{0}^{2}+\lambda_{1}^{2}}\right) s_{2}=0,
\end{aligned}
$$

and the second family of IMSMs, which corresponds to $\lambda_{2}(18)$, can be written as:

$$
\begin{aligned}
& 2 \alpha^{4} \lambda_{0}^{2} r_{1}^{2}+\left(2 \alpha^{2} \lambda_{0}^{2}+\lambda_{1}\left(\lambda_{1}+\sqrt{4 \alpha^{2} \lambda_{0}^{2}+\lambda_{1}^{2}}\right)\right) s_{2}^{2} \\
& \quad=\left(2 \alpha^{2} \lambda_{0}^{2}+\lambda_{1}\left(\lambda_{1}+\sqrt{4 \alpha^{2} \lambda_{0}^{2}+\lambda_{1}^{2}}\right)\right) \frac{\lambda_{0}}{\lambda_{3}} \\
& s_{1}^{2}+s_{2}^{2}=\frac{\lambda_{0}}{\lambda_{3}}, \quad s_{3}=0, \quad r_{3}=0, \quad 2 \alpha^{2} \lambda_{0} r_{2}+\left(\lambda_{1}+\sqrt{4 \alpha^{2} \lambda_{0}^{2}+\lambda_{1}^{2}}\right) s_{2}=0 .
\end{aligned}
$$

From the geometric viewpoint, each of these families of IMSMs - for each fixed collection of parameters of the family $\lambda_{0}, \lambda_{1}, \lambda_{3}$ - describes the curves lying in $\mathbb{R}^{6}$ at the intersection of the three hyperplanes, one elliptic "cylinder" and one circular "cylinder".

The vector field on elements of the family of IMSMs (19) is given by the differential equation:

$$
\dot{s}_{2}=-\frac{2 \alpha \lambda_{0}\left(\lambda_{0}-\lambda_{3} s_{2}^{2}\right)}{\left(\lambda_{1}+\sqrt{4 \alpha^{2} \lambda_{0}^{2}+\lambda_{1}^{2}}\right) \lambda_{3}},
$$

which is derived from equations (6) after removing $s_{1}, s_{3}, r_{1}, r_{2}, r_{3}$ from them with use of expressions (19).

The vector field on elements of the family of IMSMs (20) is given by the differential equation:

$$
\dot{s}_{2}=-\frac{2 \alpha \lambda_{0}\left(\lambda_{0}-\lambda_{3} s_{2}^{2}\right)}{\left(\lambda_{1}-\sqrt{4 \alpha^{2} \lambda_{0}^{2}+\lambda_{1}^{2}}\right) \lambda_{3}},
$$


which can also be derived from equations (6) after removing $s_{1}, s_{3}, r_{1}, r_{2}, r_{3}$ from them with the use of $(20)$.

Consider the problem of finding 2nd-level stationary solutions for the system (21) and investigation of their stability on elements of the family of IMSMs (19).

As obvious from (21), solutions of the form

$$
s_{2}^{0}=-\frac{\sqrt{\lambda_{0}}}{\sqrt{\lambda_{3}}}, \quad s_{2}^{0}=\frac{\sqrt{\lambda_{0}}}{\sqrt{\lambda_{3}}}
$$

are its stationary solutions. Now we investigate their stability by Lyapunov's method [9]. Let us consider the first of these solutions (23).

According to the above method, we consider the solution $s_{2}^{0}=-\sqrt{\lambda_{0}} / \sqrt{\lambda_{3}}$ in the capacity of undisturbed one. Next, we introduce the deviations $z=s_{2}-s_{2}^{0}$ of disturbed motion from undisturbed one, and consider the function $V=\frac{1}{2} z^{2}$ in the capacity of the Lyapunov function.

The equation of disturbed motion writes:

$$
\dot{z}=-\frac{2 \alpha \lambda_{0} z^{2}}{\lambda_{1}+\sqrt{4 \alpha^{2} \lambda_{0}^{2}+\lambda_{1}^{2}}}-\frac{4 \alpha \lambda_{0}^{3 / 2} z}{\left(\lambda_{1}+\sqrt{4 \alpha^{2} \lambda_{0}^{2}+\lambda_{1}^{2}}\right) \lambda_{3}} .
$$

The derivative of the function $V$ due to the differential equation (24) up to the 2nd order terms writes:

$$
\dot{V}=-\frac{4 \alpha \lambda_{0}^{3 / 2} z^{2}}{\left(\lambda_{1}+\sqrt{4 \alpha^{2} \lambda_{0}^{2}+\lambda_{1}^{2}}\right) \lambda_{3}}+\bar{V}_{n}
$$

where $\bar{V}_{n}$ is the $n$-order terms $(n>2)$.

The function $V$ is positive-definite. From the expression for its derivative it is obvious that it will be negative-definite when the following conditions imposed on the parameters $\lambda_{i}$ hold:

$$
\alpha>0 \vee \lambda_{0}>0 \vee\left(\lambda_{1}<0 \vee \lambda_{3}>0 \wedge \lambda_{1}>0 \vee \lambda_{3}>0\right) \text {. }
$$

According to the Lyapunov theorem [9] on stability of undisturbed motion, satisfaction of these conditions means that the solution investigated is asymptotically stable on elements of the family of IMSMs (19).

According to the above theorem, the solution under scrutiny is unstable on elements of the family of IMSMs (19) when the following conditions hold:

$$
\alpha<0 \vee \lambda_{0}>0 \vee\left(\lambda_{1}<0 \vee \lambda_{3}>0 \wedge \lambda_{1}>0 \vee \lambda_{3}>0\right) \text {. }
$$

Substitution of the stationary solution $s_{2}=-\sqrt{\lambda_{0}} / \sqrt{\lambda_{3}}$ into (19) allows one to obtain the solution corresponding to it in the whole space of variables $s_{1}, s_{2}, s_{3}, r_{1}, r_{2}, r_{3}$ :

$$
\left\{r_{1}=0, r_{2}=\frac{\lambda_{1}-\sqrt{4 \alpha^{2} \lambda_{0}^{2}+\lambda_{1}^{2}}}{2 \alpha^{2} \sqrt{\lambda_{0} \lambda_{3}}}, r_{3}=0, s_{1}=0, s_{2}=-\frac{\sqrt{\lambda_{0}}}{\sqrt{\lambda_{3}}}, s_{3}=0\right\} \text {. }
$$

Analysis of (27) showed that this solution is unstable in the sense of Lyapunov [8] under the following conditions imposed on the parameters $\lambda_{i}$ :

$$
\begin{aligned}
& \alpha<0 \vee\left(\lambda_{0}<0 \vee \lambda_{1}>0 \vee \lambda_{3}<0 \wedge \lambda_{0}>0 \vee \lambda_{1}>0 \vee \lambda_{3}>0\right) \\
& \wedge \alpha>0 \vee\left(\lambda_{0}<0 \vee \lambda_{1}>0 \vee \lambda_{3}<0 \wedge \lambda_{0}>0 \vee \lambda_{1}>0 \vee \lambda_{3}>0\right) .
\end{aligned}
$$

There are roots having positive real part among the roots of the characteristic equation constructed for the equations (6) linearized in the neighbourhood of the solution (27) when conditions (28) hold. 
Comparison of the conditions (25) and (28) shows that under the same conditions imposed on the parameters $\lambda_{i}$ :

$$
\alpha>0 \vee \lambda_{0}>0 \vee \lambda_{1}>0 \vee \lambda_{3}>0,
$$

the first of the solutions (23) asymptotically stable on the IMSM (19) corresponds to the solution which is unstable in the whole space of the problem's variables.

Similarly, comparison of the conditions (26) and (28) allows us to conclude: when the following conditions

$$
\alpha<0 \vee \lambda_{0}>0 \vee \lambda_{1}>0 \vee \lambda_{3}>0
$$

hold, the solution unstable on the IMSMs (19) corresponds to the solution unstable in the whole space of the problem's variables.

We also investigated stability of the 2nd solution of (23) and of stationary solutions of system (22). The results appeared to be similar to those given above.

As far as behaviour of the IMSMs themselves (19), (20) is concerned, note the following. The manifolds intersect and coincide under the condition $\lambda_{0}=\lambda_{1}=0$. As a result, we obtain the invariant manifold defined by the equations:

$$
s_{1}=0, \quad s_{2}=0, \quad s_{3}=0, \quad r_{3}=0 .
$$

The vector field on IMSM (29) is given by the differential equations:

$$
\dot{r}_{1}=\alpha r_{1} r_{2}, \quad \dot{r}_{2}=-\alpha^{2} r_{1}^{2} .
$$

\section{On stability of the zero solution and of IMSMs adjunct to it}

Let us consider a class of special stationary solutions. These solutions possess the following properties: several of the problem's first integrals assume stationary values on the solutions and, as a rule, there is a bifurcation of stationary solutions (invariant manifolds) of various dimensions in their neighbourhood.

For example, in completely integrable cases of the problem of rigid body's motion having one fixed point, when all the first integrals are quadratic, the manifolds of dimension 3 (it is half of the number of variables used in describing the problem) are typical IMSMs branching from special permanent rotations. Furthermore, in many cases there is the following relationship between the stability of special permanent rotations and the stability of IMSMs branching from them: branching of stable IMSMs from special permanent rotations represents the necessary and sufficient stability condition for them. If algebraic first integrals of the problem under consideration are not only quadratic then the relationship between the property of stability of special stationary solutions and the property of stability of IMSMs branching from them is more complex.

As far as Kirchhoff's equations are concerned that is obvious from their analysis in Sokolov's case, the IMSMs of both even and odd dimensions can branch from special stationary solutions (helical motions). Now, we consider a particular example of such a bifurcation of stationary solutions.

Consider the problem of stability of both the zero solution and the IMSMs adjunct to it, i.e. the stability of invariant manifolds which have at least one common point with the zero solution. Equality of the system's (9) Jacobian, which is computed for zero values of the variables $s_{i}, r_{i}$ $i=1, \ldots, 3$, to zero is the condition of existence of such IMSMs.

The Jacobian of (9) for $s_{1}=0, s_{2}=0, s_{3}=0, r_{1}=0, r_{2}=0, r_{3}=0$ writes:

$$
J=\left(\lambda_{1}^{2}+2 \lambda_{0} \lambda_{2}\right)\left(\alpha^{2} \lambda_{0}^{2}+\lambda_{1}^{2}+2 \lambda_{0} \lambda_{2}\right)\left(\alpha^{2} \lambda_{0}^{2}+\lambda_{1}^{2}+4 \lambda_{0} \lambda_{2}\right) .
$$


The following possibilities for satisfaction of the equality $J=0$ were considered (when the zero solution is special):
1) $\lambda_{0}=0, \lambda_{1}=0$
2) $\lambda_{1}=0, \lambda_{2}=0$;
3) $\lambda_{2}=-\frac{\lambda_{1}^{2}}{2 \lambda_{0}}$
4) $\lambda_{2}=-\frac{\alpha^{2} \lambda_{0}^{2}+\lambda_{1}^{2}}{2 \lambda_{0}}$
5) $\lambda_{2}=-\frac{\alpha^{2} \lambda_{0}^{2}+\lambda_{1}^{2}}{4 \lambda_{0}}$

Obtaining solutions of stationary equations (9) for the indicated values of $\lambda_{2}(30)$ with the aid of Gröbner's bases technique allowed us to find out a sufficiently large number of families of IMSMs adjunct to the zero solution. Some of these solutions are adduced below:

$$
\begin{aligned}
& \left\{s_{1}=-\frac{\lambda_{1}}{\alpha \lambda_{0}} s_{3}, s_{2}=0, r_{1}=-\frac{s_{3}}{\alpha}, r_{2}=0, r_{3}=\frac{\lambda_{0}}{\lambda_{1}} s_{3}\right\} \quad \text { for } \quad \lambda_{2}=-\frac{\alpha^{2} \lambda_{0}^{2}+\lambda_{1}^{2}}{2 \lambda_{0}} ; \\
& \left\{s_{3}=0, r_{1}=0, r_{2}=0, r_{3}=0\right\} \quad \text { for } \quad \lambda_{0}=0, \lambda_{1}=0 ; \\
& \left\{s_{1}=0, s_{2}=0, s_{3}=0, r_{1}=0, r_{3}=0\right\} \quad \text { for } \quad \lambda_{1}=0, \quad \lambda_{2}=0 ; \\
& \left\{s_{1}=0, s_{2}=\frac{\lambda_{1}}{\lambda_{0}} r_{2}, s_{3}=0, r_{1}=0, r_{2}=0\right\} \quad \text { for } \quad \lambda_{2}=-\frac{\lambda_{1}^{2}}{2 \lambda_{0}}, \lambda_{3}=0 ; \\
& \left\{s_{1}=-\frac{2 \alpha \lambda_{0} \lambda_{1}}{\alpha^{2} \lambda_{0}^{2}-\lambda_{1}^{2}} s_{3}, s_{2}=0, r_{1}=-\frac{2 \alpha \lambda_{0}^{2}}{\alpha^{2} \lambda_{0}^{2}-\lambda_{1}^{2}} s_{3}, r_{2}=0, r_{3}=-\frac{2 \alpha \lambda_{0} \lambda_{1}}{\alpha^{2} \lambda_{0}^{2}-\lambda_{1}^{2}} s_{3}\right\} \\
& \quad \text { for } \quad \lambda_{2}=-\frac{\alpha^{2} \lambda_{0}^{2}+\lambda_{1}^{2}}{4 \lambda_{0}}, \lambda_{3}=0 ; \\
& \left\{s_{1}=0, s_{2}=0, s_{3}=-\frac{\alpha}{2} r_{1}, r_{2}=0, r_{3}=0\right\} \\
& \quad \text { for } \quad \lambda_{2}=-\frac{\alpha^{2} \lambda_{0}^{2}+\lambda_{1}^{2}}{4 \lambda_{0}}, \lambda_{1}=0, \lambda_{3}=0 .
\end{aligned}
$$

These solutions represent the simplest of the invariant manifolds obtained: the zero vector field is defined on all the IMSMs (31), (32). The latter attributes some specificity to the problem of stability analysis for such manifolds.

We use the method of Lyapunov functions, in particular, the Routh-Lyapunov method, to investigate stability of the zero solution as well as the families of IMSMs (31), (32) adjunct to it.

\subsection{Investigation of stability of the zero solution}

The procedure of obtaining sufficient conditions of stability for the stationary solutions by Routh-Lyapunov's method is practically reduced to the verification (in the simplest case) of signdefiniteness of the 2nd variation of the integral $K(8)$ in the neighbourhood of the stationary solution under scrutiny. The sufficient conditions can be made "softer", if signdefiniteness of $\delta^{2} K$ is considered on the manifold defined by the first variations of each of $m-1$ integrals (where $m$ is the number of vanishing integrals in $K$ ).

Obtaining sufficient stability conditions for the zero solution by this technique is rather trivial in the computational aspect. Note only that it is stable in the sense of Lyapunov when the following restrictions imposed on the problem's parameters are satisfied:

$$
\begin{aligned}
& \alpha>0 \vee \lambda_{0}>0 \vee\left(\lambda_{1}>0 \vee \lambda_{2}<-\frac{\alpha^{2} \lambda_{0}^{2}+\lambda_{1}^{2}}{2 \lambda_{0}} \wedge \lambda_{1}<0 \vee \lambda_{2}<-\frac{\alpha^{2} \lambda_{0}^{2}+\lambda_{1}^{2}}{2 \lambda_{0}}\right) \wedge \\
& \alpha<0 \vee \lambda_{0}>0 \vee\left(\lambda_{1}>0 \vee \lambda_{2}<-\frac{\alpha^{2} \lambda_{0}^{2}+\lambda_{1}^{2}}{2 \lambda_{0}} \wedge \lambda_{1}<0 \vee \lambda_{2}<-\frac{\alpha^{2} \lambda_{0}^{2}+\lambda_{1}^{2}}{2 \lambda_{0}}\right) .
\end{aligned}
$$

It is possible to slightly "soften" the stability conditions obtained, considering that the zero solution is special. To this end, we solve the problem of choosing the "best" first integral, i.e. 
the one which gives the "most soft" sufficient stability conditions. The solution of this problem can be obtained, for example, with the use of parametric analysis of stability conditions, i.e. their minimization with respect to one or several parameters.

Consider one of the conditions imposed on the parameters (33):

$$
\lambda_{2}<-\frac{\alpha^{2} \lambda_{0}^{2}+\lambda_{1}^{2}}{2 \lambda_{0}} \quad \text { or } \quad \Lambda=2 \lambda_{2} \lambda_{0}+\alpha^{2} \lambda_{0}^{2}-\lambda_{1}^{2}<0 .
$$

Next, we find a stationary value $\Lambda$ with respect to $\lambda_{0}$ :

$$
\frac{\partial \Lambda}{\partial \lambda_{0}}=2 \lambda_{2}+2 \alpha^{2} \lambda_{0}=0
$$

The latter holds for $\lambda_{0}=-\lambda_{2} / \alpha^{2}$. Its substitution into (34) gives the following condition imposed on the parameters:

$$
-\frac{\lambda_{2}^{2}}{\alpha^{2}}-\lambda_{1}^{2}<0
$$

Hence we have stability of the equilibrium position under $\alpha \neq 0$ and $\lambda_{1} \neq 0$ or $\alpha \neq 0$ and $\lambda_{2} \neq 0$.

\subsection{Investigation of stability of IMSMs adjunct to the zero solution}

Let us investigate stability of the family of IMSMs (31). The vector field on the elements of the family of IMSMs (31) is given by the differential equation

$$
\dot{s}_{3}=0
$$

derived from equations (6) after removing $s_{1}, s_{2}, r_{1}, r_{2}, r_{3}$ from them with the aid of expressions (31).

Using (35), we may conclude that the elements of the family of IMSMs obtained represent some curves in $\mathbb{R}^{6}$, over each point of which the one-dimensional family of solutions $\left(s_{3}=s_{3}^{0}=\right.$ const) for the equation (35) is defined. Such IMSMs with the "bundle" defined on them will be called "framed invariant manifolds". Each point in the framed IMSM corresponds to some helical motion of a rigid body.

To the end of obtaining sufficient stability conditions for framed IMSMs we use the standard Lyapunov's technique.

The second variation of $K$ in the neighbourhood of some helical motion $s_{3}^{0}$, which lies on the chosen IMSM represented in terms of deviations

$$
\begin{aligned}
& z_{1}=r_{1}+\frac{s_{3}}{\alpha}, \quad z_{2}=r_{2}, \quad z_{3}=r_{3}-\frac{\lambda_{0} s_{3}}{\lambda_{1}}, \quad z_{4}=s_{1}+\frac{\lambda_{1} s_{3}}{\alpha \lambda_{0}}, \\
& z_{5}=s_{2}, \quad z_{6}=s_{3}-s_{3}^{0}
\end{aligned}
$$

writes:

$$
\begin{aligned}
\delta^{2} K= & \frac{\left(\alpha^{2} \lambda_{0}^{2}+\lambda_{1}^{2}\right)\left(\lambda_{0}-\lambda_{3} s_{3}^{0}\right)}{2 \lambda_{0}^{2}} z_{1}^{2}+\frac{\alpha^{2} \lambda_{0}^{2}+\lambda_{1}^{2}}{2 \lambda_{0}} z_{2}^{2}+\frac{\lambda_{1}^{2}}{2 \lambda_{0}} z_{3}^{2}-\lambda_{1} z_{1} z_{4}+\frac{\lambda_{0}}{2} z_{4}^{2} \\
& -\lambda_{1} z_{2} z_{5}+\frac{1}{2}\left(\lambda_{0}-\lambda_{3} s_{3}^{0^{2}}\right) z_{5}^{2} .
\end{aligned}
$$

And the respective variations of the first integrals $H, V_{1}, V_{2}$ are:

$$
\delta H=\left(\alpha z_{1}-\frac{\alpha^{2} \lambda_{0}}{\lambda_{1}} z_{3}-\frac{\lambda_{1}}{\alpha \lambda_{0}} z_{4}+\frac{\lambda_{1}^{4}-\alpha^{4} \lambda_{0}^{4}}{\alpha^{2} \lambda_{0}^{2} \lambda_{1}^{2}} z_{6}\right) s_{3}^{0}=0,
$$




$$
\begin{aligned}
& \delta V_{1}=\left(-\frac{\lambda_{1}}{\alpha \lambda_{0}} z_{1}+z_{3}-\frac{1}{\alpha} z_{4}+\frac{2\left(\alpha^{2} \lambda_{0}^{2}+\lambda_{1}^{2}\right)}{\alpha^{2} \lambda_{0} \lambda_{1}} z_{6}\right) s_{3}^{0}=0, \\
& \delta V_{2}=2\left(-\frac{1}{\alpha} z_{1}+\frac{\lambda_{0}}{\lambda_{1}} z_{3}+\frac{\alpha^{2} \lambda_{0}^{2}+\lambda_{1}^{2}}{\alpha^{2} \lambda_{1}^{2}} z_{6}\right) s_{3}^{0}=0 .
\end{aligned}
$$

Conditions of signdefiniteness for $\delta^{2} K$ are sufficient stability conditions for the elements of the family of IMSMs (31). After trivial transformations, these conditions may be written as follows:

$$
\begin{aligned}
& \lambda_{0}-\lambda_{3} s_{3}^{0^{2}}>0, \quad \alpha^{2} \lambda_{0}^{3}-\left(\alpha^{2} \lambda_{0}^{2}+\lambda_{1}^{2}\right) \lambda_{3} s_{3}^{0^{2}}>0, \\
& \lambda_{1}^{3} \lambda_{3} s_{3}^{0^{2}}+\alpha^{2} \lambda_{0}^{2} \lambda_{1}\left(\lambda_{3} s_{3}^{0^{2}}-\lambda_{0}\right) \neq 0, \quad \lambda_{0}>0, \quad \lambda_{1} \neq 0 .
\end{aligned}
$$

Now we extend the problem and investigate stability of a body's helical motions corresponding to the elements of the framed IMSM.

Assuming that $s_{3}^{0} \neq 0$, we eliminate the variables $z_{1}, z_{4}$ with the use of equations (36) (because there are only two linearly independent ones) from $\delta^{2} K$. As a result, the following quadratic form yields:

$$
\begin{aligned}
\delta^{2} \tilde{K}= & \frac{\alpha^{2} \lambda_{0}^{2}+\lambda_{1}^{2}}{2 \lambda_{0}} z_{2}^{2}+\frac{\lambda_{1}^{4}+\alpha^{2} \lambda_{0}\left(\alpha^{2} \lambda_{0}^{2}+\lambda_{1}^{2}\right)\left(\lambda_{0}-\lambda_{3} s_{3}^{0}\right)}{2 \lambda_{0} \lambda_{1}^{2}} z_{3}^{2}-\lambda_{1} z_{2} z_{5}+\frac{1}{2}\left(\lambda_{0}-\lambda_{3} s_{3}^{0^{2}}\right) z_{5}^{2} \\
& +\frac{\left(\alpha^{2} \lambda_{0}^{2}+\lambda_{1}^{2}\right)\left(\alpha^{2} \lambda_{0}^{3}-\left(\alpha^{2} \lambda_{0}^{2}+\lambda_{1}^{2}\right) \lambda_{3} s_{3}^{0}\right)}{\lambda_{0} \lambda_{1}^{3}} z_{3} z_{6} \\
& +\frac{\left(\alpha^{2} \lambda_{0}^{2}+\lambda_{1}^{2}\right)^{2}\left(\alpha^{2} \lambda_{0}^{3}-\left(\alpha^{2} \lambda_{0}^{2}+\lambda_{1}^{2}\right) \lambda_{3} s_{3}^{0^{2}}\right)}{2 \alpha^{2} \lambda_{0}^{2} \lambda_{1}^{4}} z_{6}^{2} .
\end{aligned}
$$

Conditions of signdefiniteness for $\delta^{2} \tilde{K}$ are sufficient stability ones for the helical motions, which belong to IMSMs under scrutiny. When representing them in the form of the Sylvester conditions, we have:

$$
\begin{aligned}
& \text { 1) } \frac{\alpha^{2} \lambda_{0}^{2}+\lambda_{1}^{2}}{2 \lambda_{0}}>0 \\
& \text { 2) } \frac{\left(\alpha^{2} \lambda_{0}^{2}+\lambda_{1}^{2}\right)\left(\lambda_{1}^{4}+\alpha^{2} \lambda_{0}\left(\alpha^{2} \lambda_{0}^{2}+\lambda_{1}^{2}\right)\left(\lambda_{0}-\lambda_{3} s_{3}^{0}\right)\right)}{4 \lambda_{0}^{2} \lambda_{1}^{2}}>0 \\
& \text { 3) } \frac{1}{8 \lambda_{0}^{2} \lambda_{1}^{2}}\left(\alpha^{2} \lambda_{0}^{3}-\left(\alpha^{2} \lambda_{0}^{2}+\lambda_{1}^{2}\right) \lambda_{3} s_{3}^{0}\right)\left(\lambda_{1}^{4}+\alpha^{2} \lambda_{0}\left(\alpha^{2} \lambda_{0}^{2}+\lambda_{1}^{2}\right)\left(\lambda_{0}-\lambda_{3} s_{3}^{02}\right)\right)>0 \\
& \text { 4) } \frac{\left(\alpha^{2} \lambda_{0}^{2}+\lambda_{1}^{2}\right)^{3}\left(\lambda_{1}^{2} \lambda_{3} s_{3}^{0}+\alpha^{2} \lambda_{0}^{2}\left(\lambda_{3} s_{3}^{0^{2}}-\lambda_{0}\right)\right)^{2}}{16 \alpha^{2} \lambda_{0}^{4} \lambda_{1}^{4}}>0 .
\end{aligned}
$$

A standard software package "Algebra InequalitySolve" of CAS "Mathematica" was used for the purpose of verification of compatibility for this system of inequalities. Its application to (37) showed that the inequalities are compatible when:

$$
\begin{aligned}
& \alpha<0 \vee \lambda_{0}>0 \\
& \vee\left(\lambda_{1}<0 \vee\left(\lambda_{3}<0 \wedge \lambda_{3}>0 \vee-\frac{\alpha \lambda_{0}^{3 / 2}}{\sqrt{\left(\alpha^{2} \lambda_{0}^{2}+\lambda_{1}^{2}\right) \lambda_{3}}}<s_{3}^{0}<\frac{\alpha \lambda_{0}^{3 / 2}}{\sqrt{\left(\alpha^{2} \lambda_{0}^{2}+\lambda_{1}^{2}\right) \lambda_{3}}}\right)\right. \\
& \left.\wedge \lambda_{1}>0 \vee\left(\lambda_{3}<0 \wedge \lambda_{3}>0 \vee-\frac{\alpha \lambda_{0}^{3 / 2}}{\sqrt{\left(\alpha^{2} \lambda_{0}^{2}+\lambda_{1}^{2}\right) \lambda_{3}}}<s_{3}^{0}<\frac{\alpha \lambda_{0}^{3 / 2}}{\sqrt{\left(\alpha^{2} \lambda_{0}^{2}+\lambda_{1}^{2}\right) \lambda_{3}}}\right)\right)
\end{aligned}
$$




$$
\begin{aligned}
& \wedge \alpha>0 \vee \lambda_{0}>0 \\
& \vee\left(\lambda_{1}<0 \vee\left(\lambda_{3}<0 \vee \lambda_{3}>0 \wedge-\frac{\alpha \lambda_{0}^{3 / 2}}{\sqrt{\left(\alpha^{2} \lambda_{0}^{2}+\lambda_{1}^{2}\right) \lambda_{3}}}<s_{3}^{0}<\frac{\alpha \lambda_{0}^{3 / 2}}{\sqrt{\left(\alpha^{2} \lambda_{0}^{2}+\lambda_{1}^{2}\right) \lambda_{3}}}\right)\right. \\
& \left.\wedge \lambda_{1}>0 \vee\left(\lambda_{3}<0 \vee \lambda_{3}>0 \wedge-\frac{\alpha \lambda_{0}^{3 / 2}}{\sqrt{\left(\alpha^{2} \lambda_{0}^{2}+\lambda_{1}^{2}\right) \lambda_{3}}}<s_{3}^{0}<\frac{\alpha \lambda_{0}^{3 / 2}}{\sqrt{\left(\alpha^{2} \lambda_{0}^{2}+\lambda_{1}^{2}\right) \lambda_{3}}}\right)\right) .
\end{aligned}
$$

Hence, stable in the sense of Lyapunov are only those body's helical motions for which the parameter $s_{3}^{0}$ satisfies the latter conditions.

Comparison of stability conditions for the zero solution with the stability conditions for IMSMs (31) gives evidence that satisfaction of the former conditions implies satisfaction of the latter. Consequently, the stable IMSMs adjunct to the stable zero solution.

We also investigated stability of IMSMs (32). We proved that some of these IMSMs are unstable with respect to the first approximation. Investigation of stability for other solutions necessitates involving of higher-order terms in the integral $K$ expansion. But this problem was not considered here.

\section{Conclusion}

The paper represented some results of qualitative analysis of the differential equations describing the motion of a rigid body in ideal fluid. The Kirchhoff's differential equations were considered in Sokolov's case. In this case, these have 4 algebraic first integrals (three quadratic ones and one 4th degree integral) and represent a completely integrable system.

Routh-Lyapunov's method was used to analyze the set of solutions of the equations. The method proposes a technique of finding both the stationary solutions and the invariant manifolds of motion equations, when the equations have a sufficiently large number of first integrals. The stationary solutions and invariant manifolds, which were obtained by this method, may be investigated for stability by the 2nd Lyapunov's method. Furthermore, the corresponding first integrals can be used here as Lyapunov functions.

The paper considers a rather typical case when the problem of finding stationary solutions is reduced to solving a nonlinear system of equations. Some interesting cases of stability investigation for conservative systems are also given. For example, the property of asymptotic stability of the equilibrium state for a vector field on the 1-dimensional invariant manifold of a conservative system was used for proving instability of the stationary solution (which corresponds to this equilibrium state) within the whole system's phase space. Some examples of branching invariant manifolds of various dimensions and investigation of their stability were considered.

The results of this work were obtained with the use of computer algebra tools. Such investigations cannot probably be conducted within an acceptable time without them. To ground this statement, we gave a Gröbner basis in Appendix C. This basis was constructed and used for finding solutions of a nonlinear system of algebraic equations arising in computations.

The results of qualitative analysis of Kirchhoff's differential equations represented in the paper give evidence that the technique of investigation of mechanical systems, which is based on a combination of classical methods of rigid body dynamics and computer algebra methods, is rather efficient and may be used for investigations of above type problems.

\section{A Appendix}

The following concepts were used in the paper. 
Definition 1. The solutions of differential equations, on which the first integral (an element of the algebra of problem's first integrals) assumes a stationary value, are called the stationary solutions.

This means the following. The stationary solution satisfies the equations which are the result of equating all the partial derivatives of the first integral with respect to the problem's variables to zero (stationary conditions). Such solutions simultaneously are the solutions of initial differential equations. This follows from the Lyapunov's theorem [9].

Definition 2. Manifolds, whose equations satisfy the stationary conditions for some first integral, are called the invariant manifolds of steady motions (IMSMs).

The proof of invariance of such manifolds with respect to initial differential equations can be found in Appendix B. Note, that manifolds are understood as the sets of dimension larger than zero.

If some differential equations have an IMSM then by these equations it is possible to define a vector field on this IMSM. This procedure is called a reduction of the initial system. The vector field, in turn, can have first integrals which may be used for finding stationary solutions and the 2nd-level IMSM (on the given IMSM).

Stationary solutions and manifolds are suitable in stability investigations by Lyapunov's second method, because the expansions of the corresponding first integrals in the neighbourhood of these solutions and manifolds in Taylor series do not contain linear terms.

\section{B Appendix}

More exactly, we use the following theorem:

Theorem 1. If partial derivatives of the first integral $V(x, t)$ of the system $\dot{x}_{i}=X_{i}(x, t)(i=$ $1, \ldots, n)$ with respect to the problem's phase variables have the form

$$
\frac{\partial V}{\partial x_{i}}=\sum_{l=1}^{k} a_{i l}(x, t) \varphi_{l}(x, t)+\sum_{l=1}^{k} \sum_{p=1}^{k} a_{i l}(x, t) \varphi_{l}(x, t) \varphi_{p}(x, t)+\cdots, \quad i=1, \ldots, n,
$$

and the rank of the matrix $\left\|a_{i l}(x, t)\right\|$ is " $k$ " on the manifold $\varphi_{l}(x, t)=0, l=1, \ldots, k$, then the manifold $\varphi_{l}(x, t)=0, l=1, \ldots, k$, is invariant for the initial system of differential equations.

Proof. Let the system of differential equations

$$
\dot{x}_{i}=X_{i}(x, t), \quad i=1,2, \ldots, n,
$$

have the first integral $V(x, t)$ and

$$
\frac{\partial V}{\partial x_{i}}=\sum_{l=1}^{k} a_{i l}(x, t) \varphi_{l}(x, t)+\sum_{l=1}^{k} \sum_{p=1}^{k} a_{i l p}(x, t) \varphi_{l}(x, t) \varphi_{p}(x, t)+\cdots, \quad i=1, \ldots, n .
$$

Since $V(x, t)$ is the first integral, we have

$$
\frac{d V}{d t}=\sum_{j=1}^{n} \frac{\partial V}{\partial x_{j}} X_{j}+\frac{\partial V}{\partial t}=0 .
$$

When differentiating the latter identity with respect to $x_{i}$, we obtain the system of equations

$$
\sum_{j=1}^{n} \frac{\partial}{\partial x_{i}}\left(\frac{\partial V}{\partial x_{j}}\right) X_{j}+\frac{\partial}{\partial x_{i}} \frac{\partial V}{\partial t}+\sum_{j=1}^{n} \frac{\partial V}{\partial x_{j}} \frac{\partial X_{j}}{\partial x_{i}}=0, \quad i=1, \ldots, n .
$$


Having changed the order of differentiation in the above system, we have

$$
\sum_{j=1}^{n} \frac{\partial}{\partial x_{j}}\left(\frac{\partial V}{\partial x_{i}}\right) X_{j}+\frac{\partial}{\partial t} \frac{\partial V}{\partial x_{i}}=-\sum_{j=1}^{n} \frac{\partial V}{\partial x_{j}} \frac{\partial X_{j}}{\partial x_{i}}, \quad i=1, \ldots, n .
$$

Now substitute the expression for $\partial V / \partial x_{i}$ into the latter formulae and perform differentiation with respect to $x_{i}$. After trivial transformations we have:

$$
\sum_{l=1}^{k} a_{i l}\left(\sum_{j=1}^{n} \frac{\partial \varphi_{l}}{\partial x_{j}} X_{j}+\frac{\partial \varphi_{l}}{\partial t}\right)=F_{i}\left(\varphi_{l}\right), \quad i=1, \ldots, n,
$$

where $F_{i}(0)=0$.

When $\varphi_{p}=0, p=1, \ldots, k$, the latter system transforms into the system of $n$ linear homogeneous equations:

$$
\sum_{l=1}^{k} a_{i l}\left(\sum_{j=1}^{n} \frac{\partial \varphi_{l}}{\partial x_{j}} X_{j}+\frac{\partial \varphi_{l}}{\partial t}\right)=0 \quad i=1, \ldots, n .
$$

Under the condition that the rank of the matrix $\left\|a_{i l}(x, t)\right\|$ is $k$ on the manifold $\varphi_{p}=0, p=$ $1, \ldots, k$, this system has only the following trivial solution:

$$
\sum_{j=1}^{n} \frac{\partial \varphi_{l}}{\partial x_{j}} X_{j}+\frac{\partial \varphi_{l}}{\partial t}=0, \quad l=1, \ldots, k .
$$

The latter proves that the manifold $\varphi_{p}=0, p=1, \ldots, k$ is invariant for the initial system of differential equations.

\section{Appendix}

The Gröbner basis constructed for the system (10):

$$
\begin{aligned}
& s_{2} s_{3}\left\{2 \lambda_{2}\left(\alpha^{4} \lambda_{0}^{3}+4 \alpha^{2} \lambda_{0}^{2} \lambda_{2}+2 \lambda_{2}\left(\lambda_{1}^{2}+2 \lambda_{0} \lambda_{2}\right)\right)+4 \alpha \lambda_{1} \lambda_{2}\left(\alpha^{2} \lambda_{0}+2 \lambda_{2}\right) \lambda_{3} s_{1} s_{3}\right. \\
& -2 \lambda_{2}\left(\alpha^{4} \lambda_{0}^{2}+4 \lambda_{2}^{2}-2 \alpha^{2}\left(\lambda_{1}^{2}-2 \lambda_{0} \lambda_{2}\right)\right) \lambda_{3} s_{3}^{2}+\alpha^{2}\left(\alpha^{2} \lambda_{0}+2 \lambda_{2}\right)^{2} \lambda_{3}^{2} s_{1}^{2} s_{3}^{2} \\
& \left.+2 \alpha^{3} \lambda_{1}\left(\alpha^{2} \lambda_{0}+2 \lambda_{2}\right) \lambda_{3}^{2} s_{1} s_{3}^{3}+\alpha^{4} \lambda_{1}^{2} \lambda_{3}^{2} s_{3}^{4}\right\}=0, \\
& \left(\left(\alpha^{2} \lambda_{0}+2 \lambda_{2}\right) s_{1}+\alpha \lambda_{1} s_{3}\right)\left\{2 \lambda_{2}\left(\alpha^{4} \lambda_{0}^{3}+4 \alpha^{2} \lambda_{0}^{2} \lambda_{2}+2 \lambda_{2}\left(\lambda_{1}^{2}+2 \lambda_{0} \lambda_{2}\right)\right)\right. \\
& +4 \alpha \lambda_{1} \lambda_{2}\left(\alpha^{2} \lambda_{0}+2 \lambda_{2}\right) \lambda_{3} s_{1} s_{3}-2 \lambda_{2}\left(\alpha^{4} \lambda_{0}^{2}+4 \lambda_{2}^{2}-2 \alpha^{2}\left(\lambda_{1}^{2}-2 \lambda_{0} \lambda_{2}\right)\right) \lambda_{3} s_{3}^{2} \\
& \left.+\alpha^{2}\left(\alpha^{2} \lambda_{0}+2 \lambda_{2}\right)^{2} \lambda_{3}^{2} s_{1}^{2} s_{3}^{2}+2 \alpha^{3} \lambda_{1}\left(\alpha^{2} \lambda_{0}+2 \lambda_{2}\right) \lambda_{3}^{2} s_{1} s_{3}^{3}+\alpha^{4} \lambda_{1}^{2} \lambda_{3}^{2} s_{3}^{4}\right\}=0, \\
& s_{2}\left\{2 \lambda_{2}\left(\alpha^{2} \lambda_{0}+2 \lambda_{2}\right)^{3}\left(\lambda_{1}^{2}+2 \lambda_{0} \lambda_{2}\right)+4 \alpha^{2} \lambda_{0} \lambda_{2}\left(\alpha^{2} \lambda_{0}+2 \lambda_{2}\right)\left(\alpha^{2} \lambda_{0}-\alpha \lambda_{1}+2 \lambda_{2}\right)\right. \\
& \times\left(\alpha\left(\alpha \lambda_{0}+\lambda_{1}\right)+2 \lambda_{2}\right) \lambda_{3} s_{1}^{2}-\alpha^{4} \lambda_{0}\left(\alpha^{2} \lambda_{0}+2 \lambda_{2}\right)^{3} \lambda_{3}^{2} s_{1}^{4}+4 \alpha^{2} \lambda_{0} \lambda_{2}\left(\alpha^{2} \lambda_{0}+2 \lambda_{2}\right)^{3} \lambda_{3} s_{2}^{2} \\
& +\alpha^{4} \lambda_{0}\left(\alpha^{2} \lambda_{0}+2 \lambda_{2}\right)^{3} \lambda_{3}^{2} s_{2}^{4}+4 \alpha \lambda_{1} \lambda_{2}\left(2 \alpha^{6} \lambda_{0}^{3}+16 \alpha^{2} \lambda_{0} \lambda_{2}^{2}+8 \lambda_{2}^{3}\right. \\
& \left.+\alpha^{4} \lambda_{0}\left(10 \lambda_{0} \lambda_{2}-\lambda_{1}^{2}\right)\right) \lambda_{3} s_{1} s_{3}-4 \alpha^{5} \lambda_{0} \lambda_{1}\left(\alpha^{2} \lambda_{0}+2 \lambda_{2}\right)^{2} \lambda_{3}^{2} s_{1}^{3} s_{3}-2 \lambda_{2}\left(\alpha^{2} \lambda_{0}+2 \lambda_{2}\right) \\
& \times\left(8 \lambda_{2}^{3}-4 \alpha^{2} \lambda_{2}\left(\lambda_{1}^{2}-2 \lambda_{0} \lambda_{2}\right)+\alpha^{4} \lambda_{0}\left(2 \lambda_{0} \lambda_{2}-\lambda_{1}^{2}\right)\right) \lambda_{3} s_{3}^{2}+\alpha^{2}\left(\alpha^{2} \lambda_{0}+2 \lambda_{2}\right) \\
& \times\left(8 \alpha^{2} \lambda_{0} \times \lambda_{2}^{2}+8 \lambda_{2}^{3}+\alpha^{4} \lambda_{0}\left(-5 \lambda_{1}^{2}+2 \lambda_{0} \lambda_{2}\right)\right) \lambda_{3}^{2} s_{1}^{2} s_{3}^{2}+2 \alpha^{3} \lambda_{1}\left(8 \alpha^{2} \lambda_{0} \lambda_{2}^{2}+8 \lambda_{2}^{3}\right. \\
& \left.\left.\times+\alpha^{4} \lambda_{0}\left(2 \lambda_{0} \lambda_{2}-\lambda_{1}^{2}\right)\right) \lambda_{3}^{2} s_{1} s_{3}^{3}+2 \alpha^{4} \lambda_{1}^{2} \lambda_{2}\left(\alpha^{2} \lambda_{0}+2 \lambda_{2}\right) \lambda_{3}^{2} s_{3}^{4}\right\}=0, \\
& s_{3}\left\{2 \lambda_{2}\left(\alpha^{4} \lambda_{0}^{3}+4 \alpha^{2} \lambda_{0}^{2} \lambda_{2}+2 \lambda_{2}\left(\lambda_{1}^{2}+2 \lambda_{0} \lambda_{2}\right)\right)+4 \alpha \lambda_{1} \lambda_{2}\left(\alpha^{2} \lambda_{0}+2 \lambda_{2}\right) \lambda_{3} s_{1} s_{3}\right.
\end{aligned}
$$




$$
\begin{aligned}
& -2 \lambda_{2}\left(\alpha^{4} \lambda_{0}^{2}+4 \lambda_{2}^{2}-2 \alpha^{2}\left(\lambda_{1}^{2}-2 \lambda_{0} \lambda_{2}\right)\right) \lambda_{3} s_{3}^{2}+\alpha^{2}\left(\alpha^{2} \lambda_{0}+2 \lambda_{2}\right)^{2} \lambda_{3}^{2} s_{1}^{2} s_{3}^{2} \\
& \left.+2 \alpha^{3} \lambda_{1}\left(\alpha^{2} \lambda_{0}+2 \lambda_{2}\right) \lambda_{3}^{2} s_{1} s_{3}^{3}+\alpha^{4} \lambda_{1}^{2} \lambda_{3}^{2} s_{3}^{4}\right\}\left\{\left(\alpha^{2} \lambda_{0}+2 \lambda_{2}\right)^{4}\left(\alpha^{2} \lambda_{0}^{2}+\lambda_{1}^{2}+4 \lambda_{0} \lambda_{2}\right)\right. \\
& -4 \lambda_{2}\left(\alpha^{2} \lambda_{0}+2 \lambda_{2}\right)^{2}\left(\alpha^{4} \lambda_{0}^{2}+4 \lambda_{2}^{2}+\alpha^{2}\left(\lambda_{1}^{2}+4 \lambda_{0} \lambda_{2}\right)\right) \lambda_{3} s_{3}^{2}-\alpha^{2}\left(\alpha^{4} \lambda_{0}^{2}+4 \lambda_{2}^{2}\right. \\
& \left.\left.+\alpha^{2}\left(\lambda_{1}^{2}+4 \lambda_{0} \lambda_{2}\right)\right)^{2} \lambda_{3}^{2} s_{3}^{4}\right\}=0, \\
& 2 \lambda_{1} \lambda_{2}\left(\alpha^{2} \lambda_{0}+2 \lambda_{2}\right)^{2} r_{2}-2 \lambda_{0} \lambda_{2}\left(\alpha^{2} \lambda_{0}+2 \lambda_{2}\right)^{2} s_{2}-\lambda_{0}\left(\alpha^{3} \lambda_{0}+2 \alpha \lambda_{2}\right)^{2} \lambda_{3} s_{1}^{2} s_{2} \\
& -\lambda_{0}\left(\alpha^{3} \lambda_{0}+2 \alpha \lambda_{2}\right)^{2} \lambda_{3} s_{2}^{3}-\alpha \lambda_{1}\left(\alpha^{2} \lambda_{0}+2 \lambda_{2}\right)^{2} \lambda_{3} s_{1} s_{2} s_{3}+2 \lambda_{2}\left(\alpha^{2} \lambda_{0}-\alpha \lambda_{1}+2 \lambda_{2}\right) \\
& \times\left(\alpha\left(\alpha \lambda_{0}+\lambda_{1}\right)+2 \lambda_{2}\right) \lambda_{3} s_{2} s_{3}^{2}-\alpha^{2}\left(\alpha^{2} \lambda_{0}+2 \lambda_{2}\right)^{2} \lambda_{3}^{2} s_{1}^{2} s_{2} s_{3}^{2} \\
& -2 \alpha^{3} \lambda_{1}\left(\alpha^{2} \lambda_{0}+2 \lambda_{2}\right) \lambda_{3}^{2} s_{1} s_{2} s_{3}^{3}-\alpha^{4} \lambda_{1}^{2} \lambda_{3}^{2} s_{2} s_{3}^{4}=0, \\
& s_{3}\left\{-2 \lambda_{2}\left(\alpha^{2} \lambda_{0}+2 \lambda_{2}\right)^{8}\left(\alpha^{2} \lambda_{0}^{2}+\lambda_{1}^{2}+4 \lambda_{0} \lambda_{2}\right)+\left(\alpha^{2} \lambda_{0}+2 \lambda_{2}\right)^{2}\left(\alpha^{16} \lambda_{0}^{8}+256 \lambda_{2}^{8}\right.\right. \\
& +16 \alpha^{12} \lambda_{0}^{5} \lambda_{2}\left(\lambda_{1}^{2}+7 \lambda_{0} \lambda_{2}\right)+\alpha^{14} \lambda_{0}^{6}\left(\lambda_{1}^{2}+16 \lambda_{0} \lambda_{2}\right)+64 \alpha^{2} \lambda_{2}^{6}\left(3 \lambda_{1}^{2}+16 \lambda_{0} \lambda_{2}\right) \\
& +32 \alpha^{4} \lambda_{2}^{4}\left(\lambda_{1}^{4}+16 \lambda_{0} \lambda_{1}^{2} \lambda_{2}+56 \lambda_{0}^{2} \lambda_{2}^{2}\right)+4 \alpha^{10} \lambda_{0}^{3} \lambda_{2}\left(\lambda_{1}^{4}+25 \lambda_{0} \lambda_{1}^{2} \lambda_{2}+112 \lambda_{0}^{2} \lambda_{2}^{2}\right) \\
& +8 \alpha^{8} \lambda_{0}^{2} \lambda_{2}^{2}\left(3 \lambda_{1}^{4}+40 \lambda_{0} \lambda_{1}^{2} \lambda_{2}+140 \lambda_{0}^{2} \lambda_{2}^{2}\right)+4 \alpha^{6} \lambda_{2}^{2}\left(\lambda_{1}^{6}+12 \lambda_{0} \lambda_{1}^{4} \lambda_{2}+140 \lambda_{0}^{2} \lambda_{1}^{2} \lambda_{2}^{2}\right. \\
& \left.\left.+448 \lambda_{0}^{3} \lambda_{2}^{3}\right)\right) \lambda_{3} s_{1}^{2}+\left(\alpha^{2} \lambda_{0}+2 \lambda_{2}\right)^{2}\left(\alpha^{16} \lambda_{0}^{8}+256 \lambda_{2}^{8}+16 \alpha^{12} \lambda_{0}^{5} \lambda_{2}\left(\lambda_{1}^{2}+7 \lambda_{0} \lambda_{2}\right)\right. \\
& +\alpha^{14} \lambda_{0}^{6}\left(\lambda_{1}^{2}+16 \lambda_{0} \lambda_{2}\right)+64 \alpha^{2} \lambda_{2}^{6}\left(3 \lambda_{1}^{2}+16 \lambda_{0} \lambda_{2}\right)+32 \alpha^{4} \lambda_{2}^{4}\left(\lambda_{1}^{4}+16 \lambda_{0} \lambda_{1}^{2} \lambda_{2}+56 \lambda_{0}^{2} \lambda_{2}^{2}\right) \\
& +4 \alpha^{10} \lambda_{0}^{3} \lambda_{2}\left(\lambda_{1}^{4}+25 \lambda_{0} \lambda_{1}^{2} \lambda_{2}+112 \lambda_{0}^{2} \lambda_{2}^{2}\right)+8 \alpha^{8} \lambda_{0}^{2} \lambda_{2}^{2}\left(3 \lambda_{1}^{4}+40 \lambda_{0} \lambda_{1}^{2} \lambda_{2}+140 \lambda_{0}^{2} \lambda_{2}^{2}\right) \\
& \left.+4 \alpha^{6} \lambda_{2}^{2}\left(\lambda_{1}^{6}+12 \lambda_{0} \lambda_{1}^{4} \lambda_{2}+140 \lambda_{0}^{2} \lambda_{1}^{2} \lambda_{2}^{2}+448 \lambda_{0}^{3} \lambda_{2}^{3}\right)\right) \lambda_{3} s_{2}^{2}+2 \alpha \lambda_{1}\left(\alpha^{2} \lambda_{0}+2 \lambda_{2}\right)^{3} \\
& \times\left(\alpha^{12} \lambda_{0}^{6}-96 \alpha^{2} \lambda_{0} \lambda_{2}^{5}-64 \lambda_{2}^{6}-16 \alpha^{4} \lambda_{0} \lambda_{2}^{3}\left(\lambda_{0} \lambda_{2}-\lambda_{1}^{2}\right)+2 \alpha^{6} \lambda_{0} \lambda_{2}\left(\lambda_{1}^{2}+4 \lambda_{0} \lambda_{2}\right)\right. \\
& \left.\times\left(\lambda_{1}^{2}+6 \lambda_{0} \lambda_{2}\right)+4 \alpha^{8} \lambda_{0}^{3} \lambda_{2}\left(2 \lambda_{1}^{2}+9 \lambda_{0} \lambda_{2}\right)+\alpha^{10} \lambda_{0}^{4}\left(\lambda_{1}^{2}+10 \lambda_{0} \lambda_{2}\right)\right) \lambda_{3} s_{1} s_{3} \\
& +\left(\alpha^{18} \lambda_{0}^{8} \lambda_{1}^{2}+2048 \lambda_{2}^{1} 0-256 \alpha^{2} \lambda_{2}^{8}\left(\lambda_{1}^{2}-32 \lambda_{0} \lambda_{2}\right)+\alpha^{16} \lambda_{0}^{6}\left(\lambda_{1}^{4}+12 \lambda_{0} \lambda_{1}^{2} \lambda_{2}+8 \lambda_{0}^{2} \lambda_{2}^{2}\right)\right. \\
& +8 \alpha^{14} \lambda_{0}^{5} \lambda_{2}\left(\lambda_{1}^{4}+7 \lambda_{0} \lambda_{1}^{2} \lambda_{2}+16 \lambda_{0}^{2} \lambda_{2}^{2}\right)+64 \alpha^{4} \lambda_{2}^{6}\left(224 \lambda_{0}^{2} \lambda_{2}^{2}-3 \lambda_{1}^{4}-12 \lambda_{0} \lambda_{1}^{2} \lambda_{2}\right) \\
& +4 \alpha^{12} \lambda_{0}^{4} \lambda_{2}^{2}\left(3 \lambda_{1}^{4}+28 \lambda_{0} \lambda_{1}^{2} \lambda_{2}+224 \lambda_{0}^{2} \lambda_{2}^{2}\right)-32 \alpha^{6} \lambda_{2}^{4}\left(\lambda_{1}^{6}+12 \lambda_{0} \lambda_{1}^{4} \lambda_{2}+28 \lambda_{0}^{2} \lambda_{1}^{2} \lambda_{2}^{2}\right. \\
& \left.-448 \lambda_{0}^{3} \lambda_{2}^{3}\right)+8 \alpha^{10} \lambda_{0}^{2} \lambda_{2}^{2}\left(448 \lambda_{0}^{3} \lambda_{2}^{3}-\lambda_{1}^{6}-8 \lambda_{0} \lambda_{1}^{4} \lambda_{2}\right)-4 \alpha^{8} \lambda_{2}^{2}\left(\lambda_{1}^{8}+8 \lambda_{0} \lambda_{1}^{6} \lambda_{2}+68 \lambda_{0}^{2} \lambda_{1}^{4} \lambda_{2}^{2}\right. \\
& \left.\left.+112 \lambda_{0}^{3} \lambda_{1}^{2} \lambda_{2}^{3}-2240 \lambda_{0}^{4} \lambda_{2}^{4}\right)\right) \lambda_{3} s_{3}^{2}-\alpha^{2}\left(\alpha^{2} \lambda_{0}+2 \lambda_{2}\right)^{2}\left(\alpha^{4} \lambda_{0}^{2}+4 \lambda_{2}^{2}+\alpha^{2}\left(\lambda_{1}^{2}+4 \lambda_{0} \lambda_{2}\right)\right) \\
& \times\left(\alpha^{10} \lambda_{0}^{5}+64 \lambda_{2}^{5}+\alpha^{8} \lambda_{0}^{3}\left(\lambda_{1}^{2}+12 \lambda_{0} \lambda_{2}\right)+8 \alpha^{2} \lambda_{2}^{3}\left(\lambda_{1}^{2}+18 \lambda_{0} \lambda_{2}\right)+2 \alpha^{6} \lambda_{0}^{2} \lambda_{2}\left(3 \lambda_{1}^{2}\right.\right. \\
& \left.\left.+28 \lambda_{0} \lambda_{2}\right)+2 \alpha^{4} \lambda_{2}\left(\lambda_{1}^{4}+6 \lambda_{0} \lambda_{1}^{2} \lambda_{2}+64 \lambda_{0}^{2} \lambda_{2}^{2}\right)\right) \lambda_{3}^{2} s_{1}^{2} s_{3}^{2}-2 \alpha^{3} \lambda_{1}\left(\alpha^{2} \lambda_{0}+2 \lambda_{2}\right)\left(\alpha^{4} \lambda_{0}^{2}+4 \lambda_{2}^{2}\right. \\
& \left.+\alpha^{2}\left(\lambda_{1}^{2}+4 \lambda_{0} \lambda_{2}\right)\right)\left(\alpha^{10} \lambda_{0}^{5}+96 \lambda_{2}^{5}+8 \alpha^{6} \lambda_{0}^{2} \lambda_{2}\left(\lambda_{1}^{2}+9 \lambda_{0} \lambda_{2}\right)+16 \alpha^{2} \lambda_{2}^{3}\left(\lambda_{1}^{2}+13 \lambda_{0} \lambda_{2}\right)\right. \\
& \left.+\alpha^{8} \lambda_{0}^{3}\left(\lambda_{1}^{2}+14 \lambda_{0} \lambda_{2}\right)+2 \alpha^{4} \lambda_{2}\left(\lambda_{1}^{4}+10 \lambda_{0} \lambda_{1}^{2} \lambda_{2}+88 \lambda_{0}^{2} \lambda_{2}^{2}\right)\right) \lambda_{3}^{2} s_{1} s_{3}^{3}+\alpha^{2}\left(\alpha^{4} \lambda_{0}^{2}+4 \lambda_{2}^{2}\right. \\
& \left.+\alpha^{2}\left(\lambda_{1}^{2}+4 \lambda_{0} \lambda_{2}\right)\right)\left(128 \lambda_{2}^{7}-96 \alpha^{2} \lambda_{2}^{5}\left(\lambda_{1}^{2}-4 \lambda_{0} \lambda_{2}\right)+\alpha^{12} \lambda_{0}^{5}\left(2 \lambda_{0} \lambda_{2}-\lambda_{1}^{2}\right)\right. \\
& +\alpha^{10} \lambda_{0}^{3}\left(24 \lambda_{0}^{2} \lambda_{2}^{2}-\lambda_{1}^{4}-14 \lambda_{0} \lambda_{1}^{2} \lambda_{2}\right)+2 \alpha^{8} \lambda_{0}^{2} \lambda_{2}\left(60 \lambda_{0}^{2} \lambda_{2}^{2}-5 \lambda_{1}^{4}-36 \lambda_{0} \lambda_{1}^{2} \lambda_{2}\right) \\
& +8 \alpha^{4} \lambda_{2}^{3}\left(60 \lambda_{0}^{2} \lambda_{2}^{2}-3 \lambda_{1}^{4}-26 \lambda_{0} \lambda_{1}^{2} \lambda_{2}\right)-2 \alpha^{6} \lambda_{2}\left(\lambda_{1}^{6}+14 \lambda_{0} \lambda_{1}^{4} \lambda_{2}+88 \lambda_{0}^{2} \lambda_{1}^{2} \lambda_{2}^{2}\right. \\
& \left.\left.-160 \lambda_{0}^{3} \lambda_{2}^{3}\right)\right) \lambda_{3}^{2} s_{3}^{4}-\left(\alpha^{3} \lambda_{0}+2 \alpha \lambda_{2}\right)^{4}\left(\alpha^{4} \lambda_{0}^{2}+4 \lambda_{2}^{2}+\alpha^{2}\left(\lambda_{1}^{2}+4 \lambda_{0} \lambda_{2}\right)\right)^{2} \lambda_{3}^{3} s_{1}^{2} s_{3}^{4} \\
& -2 \alpha^{5} \lambda_{1}\left(\alpha^{2} \lambda_{0}+2 \lambda_{2}\right)^{3}\left(\alpha^{4} \lambda_{0}^{2}+4 \lambda_{2}^{2}+\alpha^{2}\left(\lambda_{1}^{2}+4 \lambda_{0} \lambda_{2}\right)\right)^{2} \lambda_{3}^{3} s_{1} s_{3}^{5}-\alpha^{6} \lambda_{1}^{2}\left(\alpha^{2} \lambda_{0}+2 \lambda_{2}\right)^{2} \\
& \left.\times\left(\alpha^{4} \lambda_{0}^{2}+4 \lambda_{2}^{2}+\alpha^{2}\left(\lambda_{1}^{2}+4 \lambda_{0} \lambda_{2}\right)\right)^{2} \lambda_{3}^{3} s_{3}^{6}\right\}=0, \\
& -2 \lambda_{1}^{2} \lambda_{2}\left(\alpha^{2} \lambda_{0}+2 \lambda_{2}\right)\left(\alpha^{16} \lambda_{0}^{8}+256 \lambda_{2}^{8}+16 \alpha^{12} \lambda_{0}^{5} \lambda_{2}\left(\lambda_{1}^{2}+7 \lambda_{0} \lambda_{2}\right)+\alpha^{14} \lambda_{0}^{6}\left(\lambda_{1}^{2}+16 \lambda_{0} \lambda_{2}\right)\right. \\
& +64 \alpha^{2} \lambda_{2}^{6}\left(3 \lambda_{1}^{2}+16 \lambda_{0} \lambda_{2}\right)+32 \alpha^{4} \lambda_{2}^{4}\left(\lambda_{1}^{4}+16 \lambda_{0} \lambda_{1}^{2} \lambda_{2}+56 \lambda_{0}^{2} \lambda_{2}^{2}\right) \\
& +4 \alpha^{10} \lambda_{0}^{3} \lambda_{2}\left(\lambda_{1}^{4}+25 \lambda_{0} \lambda_{1}^{2} \lambda_{2}+112 \lambda_{0}^{2} \lambda_{2}^{2}\right)+8 \alpha^{8} \lambda_{0}^{2} \lambda_{2}^{2}\left(3 \lambda_{1}^{4}+40 \lambda_{0} \lambda_{1}^{2} \lambda_{2}+140 \lambda_{0}^{2} \lambda_{2}^{2}\right) \\
& \left.+4 \alpha^{6} \lambda_{2}^{2}\left(\lambda_{1}^{6}+12 \lambda_{0} \lambda_{1}^{4} \lambda_{2}+140 \lambda_{0}^{2} \lambda_{1}^{2} \lambda_{2}^{2}+448 \lambda_{0}^{3} \lambda_{2}^{3}\right)\right) s_{1}-\left(\alpha^{2} \lambda_{0}+2 \lambda_{2}\right)^{3}\left(\alpha^{16} \lambda_{0}^{8}+256 \lambda_{2}^{8}\right. \\
& +16 \alpha^{12} \lambda_{0}^{5} \lambda_{2}\left(\lambda_{1}^{2}+7 \lambda_{0} \lambda_{2}\right)+\alpha^{14} \lambda_{0}^{6}\left(\lambda_{1}^{2}+16 \lambda_{0} \lambda_{2}\right)+64 \alpha^{2} \lambda_{2}^{6}\left(3 \lambda_{1}^{2}+16 \lambda_{0} \lambda_{2}\right)
\end{aligned}
$$




$$
\begin{aligned}
& +32 \alpha^{4} \lambda_{2}^{4}\left(\lambda_{1}^{4}+16 \lambda_{0} \lambda_{1}^{2} \lambda_{2}+56 \lambda_{0}^{2} \lambda_{2}^{2}\right)+4 \alpha^{10} \lambda_{0}^{3} \lambda_{2}\left(\lambda_{1}^{4}+25 \lambda_{0} \lambda_{1}^{2} \lambda_{2}+112 \lambda_{0}^{2} \lambda_{2}^{2}\right) \\
& +8 \alpha^{8} \lambda_{0}^{2} \lambda_{2}^{2}\left(3 \lambda_{1}^{4}+40 \lambda_{0} \lambda_{1}^{2} \lambda_{2}+140 \lambda_{0}^{2} \lambda_{2}^{2}\right)+4 \alpha^{6} \lambda_{2}^{2}\left(\lambda_{1}^{6}+12 \lambda_{0} \lambda_{1}^{4} \lambda_{2}+140 \lambda_{0}^{2} \lambda_{1}^{2} \lambda_{2}^{2}\right. \\
& \left.\left.+448 \lambda_{0}^{3} \lambda_{2}^{3}\right)\right) \lambda_{3} s_{1}^{3}-\left(\alpha^{2} \lambda_{0}+2 \lambda_{2}\right)^{3}\left(\alpha^{16} \lambda_{0}^{8}+256 \lambda_{2}^{8}+16 \alpha^{12} \lambda_{0}^{5} \lambda_{2}\left(\lambda_{1}^{2}+7 \lambda_{0} \lambda_{2}\right)\right. \\
& +\alpha^{14} \lambda_{0}^{6}\left(\lambda_{1}^{2}+16 \lambda_{0} \lambda_{2}\right)+64 \alpha^{2} \lambda_{2}^{6}\left(3 \lambda_{1}^{2}+16 \lambda_{0} \lambda_{2}\right)+32 \alpha^{4} \lambda_{2}^{4}\left(\lambda_{1}^{4}+16 \lambda_{0} \lambda_{1}^{2} \lambda_{2}+56 \lambda_{0}^{2} \lambda_{2}^{2}\right) \\
& +4 \alpha^{10} \lambda_{0}^{3} \lambda_{2}\left(\lambda_{1}^{4}+25 \lambda_{0} \lambda_{1}^{2} \lambda_{2}+112 \lambda_{0}^{2} \lambda_{2}^{2}\right)+8 \alpha^{8} \lambda_{0}^{2} \lambda_{2}^{2}\left(3 \lambda_{1}^{4}+40 \lambda_{0} \lambda_{1}^{2} \lambda_{2}+140 \lambda_{0}^{2} \lambda_{2}^{2}\right) \\
& \left.+4 \alpha^{6} \lambda_{2}^{2}\left(\lambda_{1}^{6}+12 \lambda_{0} \lambda_{1}^{4} \times \lambda_{2}+140 \lambda_{0}^{2} \lambda_{1}^{2} \lambda_{2}^{2}+448 \lambda_{0}^{3} \lambda_{2}^{3}\right)\right) \lambda_{3} s_{1} s_{2}^{2}-2 \alpha \lambda_{1} \lambda_{2}\left(\alpha^{4} \lambda_{0}^{3}\right. \\
& \left.+4 \alpha^{2} \lambda_{0}^{2} \lambda_{2}+2 \lambda_{2}\left(\lambda_{1}^{2}+2 \lambda_{0} \lambda_{2}\right)\right)\left(\alpha^{4} \lambda_{0}^{2}+4 \lambda_{2}^{2}+\alpha^{2}\left(\lambda_{1}^{2}+4 \lambda_{0} \lambda_{2}\right)\right)\left(\alpha^{10} \lambda_{0}^{5}+64 \lambda_{2}^{5}\right. \\
& +\alpha^{8} \lambda_{0}^{3}\left(\lambda_{1}^{2}+12 \lambda_{0} \lambda_{2}\right)+8 \alpha^{2} \lambda_{2}^{3}\left(\lambda_{1}^{2}+18 \lambda_{0} \lambda_{2}\right)+2 \alpha^{6} \lambda_{0}^{2} \lambda_{2}\left(3 \lambda_{1}^{2}+28 \lambda_{0} \lambda_{2}\right) \\
& \left.+2 \alpha^{4} \lambda_{2}\left(\lambda_{1}^{4}+6 \lambda_{0} \lambda_{1}^{2} \lambda_{2}+64 \lambda_{0}^{2} \lambda_{2}^{2}\right)\right) s_{3}-2 \alpha \lambda_{1}\left(\alpha^{2} \lambda_{0}+2 \lambda_{2}\right)^{2}\left(\alpha^{16} \lambda_{0}^{8}+256 \lambda_{2}^{8}\right. \\
& +16 \alpha^{12} \lambda_{0}^{5} \lambda_{2}\left(\lambda_{1}^{2}+7 \lambda_{0} \lambda_{2}\right)+\alpha^{14} \lambda_{0}^{6}\left(\lambda_{1}^{2}+16 \lambda_{0} \lambda_{2}\right)+64 \alpha^{2} \lambda_{2}^{6}\left(3 \lambda_{1}^{2}+16 \lambda_{0} \lambda_{2}\right) \\
& +32 \alpha^{4} \lambda_{2}^{4}\left(\lambda_{1}^{4}+16 \lambda_{0} \lambda_{1}^{2} \lambda_{2}+56 \lambda_{0}^{2} \lambda_{2}^{2}\right)+4 \alpha^{10} \lambda_{0}^{3} \lambda_{2}\left(\lambda_{1}^{4}+25 \lambda_{0} \lambda_{1}^{2} \lambda_{2}+112 \lambda_{0}^{2} \lambda_{2}^{2}\right) \\
& +8 \alpha^{8} \lambda_{0}^{2} \lambda_{2}^{2}\left(3 \lambda_{1}^{4}+40 \lambda_{0} \lambda_{1}^{2} \lambda_{2}+140 \lambda_{0}^{2} \lambda_{2}^{2}\right)+4 \alpha^{6} \lambda_{2}^{2}\left(\lambda_{1}^{6}+12 \lambda_{0} \lambda_{1}^{4} \lambda_{2}+140 \lambda_{0}^{2} \lambda_{1}^{2} \lambda_{2}^{2}\right. \\
& \left.\left.+448 \lambda_{0}^{3} \lambda_{2}^{3}\right)\right) \lambda_{3} s_{1}^{2} s_{3}-\alpha^{2} \lambda_{1}^{2}\left(\alpha^{2} \lambda_{0}+2 \lambda_{2}\right)\left(\alpha^{16} \lambda_{0}^{8}+1280 \lambda_{2}^{8}+\alpha^{14} \lambda_{0}^{6}\left(\lambda_{1}^{2}+20 \lambda_{0} \lambda_{2}\right)\right. \\
& +8 \alpha^{12} \lambda_{0}^{5} \lambda_{2}\left(3 \lambda_{1}^{2}+22 \lambda_{0} \lambda_{2}\right)+64 \alpha^{2} \lambda_{2}^{6}\left(9 \lambda_{1}^{2}+68 \lambda_{0} \lambda_{2}\right)+32 \alpha^{4} \lambda_{2}^{4}\left(3 \lambda_{1}^{4}+44 \lambda_{0} \lambda_{1}^{2} \lambda_{2}\right. \\
& \left.+200 \lambda_{0}^{2} \lambda_{2}^{2}\right)+4 \alpha^{10} \lambda_{0}^{3} \lambda_{2}\left(2 \lambda_{1}^{4}+47 \lambda_{0} \lambda_{1}^{2} \lambda_{2}+220 \lambda_{0}^{2} \lambda_{2}^{2}\right)+8 \alpha^{8} \lambda_{0}^{2} \lambda_{2}^{2}\left(7 \lambda_{1}^{4}+88 \lambda_{0} \lambda_{1}^{2} \lambda_{2}\right. \\
& \left.\left.+340 \lambda_{0}^{2} \lambda_{2}^{2}\right)+4 \alpha^{6} \lambda_{2}^{2}\left(3 \lambda_{1}^{6}+32 \lambda_{0} \lambda_{1}^{4} \lambda_{2}+348 \lambda_{0}^{2} \lambda_{1}^{2} \lambda_{2}^{2}+1328 \lambda_{0}^{3} \lambda_{2}^{3}\right)\right) \lambda_{3} s_{1} s_{3}^{2} \\
& +4 \alpha \lambda_{1} \lambda_{2}\left(\alpha^{4} \lambda_{0}^{2}+4 \lambda_{2}^{2}+\alpha^{2}\left(\lambda_{1}^{2}+4 \lambda_{0} \lambda_{2}\right)\right)\left(128 \lambda_{2}^{7}-64 \alpha^{2} \lambda_{2}^{5}\left(\lambda_{1}^{2}-6 \lambda_{0} \lambda_{2}\right)\right. \\
& +\alpha^{12} \lambda_{0}^{5}\left(2 \lambda_{0} \lambda_{2}-\lambda_{1}^{2}\right)-8 \alpha^{4} \lambda_{2}^{3}\left(\lambda_{1}^{4}+18 \lambda_{0} \lambda_{1}^{2} \lambda_{2}-60 \lambda_{0}^{2} \lambda_{2}^{2}\right)+\alpha^{10} \lambda_{0}^{3}\left(24 \lambda_{0}^{2} \lambda_{2}^{2}-\lambda_{1}^{4}\right. \\
& \left.-12 \lambda_{0} \lambda_{1}^{2} \lambda_{2}\right)+2 \alpha^{8} \lambda_{0}^{2} \lambda_{2}\left(60 \lambda_{0}^{2} \lambda_{2}^{2}-3 \lambda_{1}^{4}-28 \lambda_{0} \lambda_{1}^{2} \lambda_{2}\right)-2 \alpha^{6} \lambda_{2}\left(\lambda_{1}^{2}-2 \lambda_{0} \lambda_{2}\right) \\
& \left.\times\left(\lambda_{1}^{4}+8 \lambda_{0} \lambda_{1}^{2} \lambda_{2}+80 \lambda_{0}^{2} \lambda_{2}^{2}\right)\right) \lambda_{3} s_{3}^{3}-\alpha^{3} \lambda_{1}\left(\alpha^{2} \lambda_{0}+2 \lambda_{2}\right)^{2}\left(\alpha^{4} \lambda_{0}^{2}+4 \lambda_{2}^{2}\right. \\
& \left.+\alpha^{2}\left(\lambda_{1}^{2}+4 \lambda_{0} \lambda_{2}\right)\right)\left(\alpha^{10} \lambda_{0}^{5}+64 \lambda_{2}^{5}+\alpha^{8} \lambda_{0}^{3}\left(\lambda_{1}^{2}+12 \lambda_{0} \lambda_{2}\right)+8 \alpha^{2} \lambda_{2}^{3}\left(\lambda_{1}^{2}+18 \lambda_{0} \lambda_{2}\right)\right. \\
& \left.+2 \alpha^{6} \lambda_{0}^{2} \lambda_{2}\left(3 \lambda_{1}^{2}+28 \lambda_{0} \lambda_{2}\right)+2 \alpha^{4} \lambda_{2}\left(\lambda_{1}^{4}+6 \lambda_{0} \lambda_{1}^{2} \lambda_{2}+64 \lambda_{0}^{2} \lambda_{2}^{2}\right)\right) \lambda_{3}^{2} s_{1}^{2} s_{3}^{3} \\
& -2 \alpha^{4} \lambda_{1}^{2}\left(\alpha^{2} \lambda_{0}+2 \lambda_{2}\right)\left(\alpha^{4} \lambda_{0}^{2}+4 \lambda_{2}^{2}+\alpha^{2}\left(\lambda_{1}^{2}+4 \lambda_{0} \lambda_{2}\right)\right)\left(\alpha^{10} \lambda_{0}^{5}+96 \lambda_{2}^{5}\right. \\
& +8 \alpha^{6} \lambda_{0}^{2} \lambda_{2}\left(\lambda_{1}^{2}+9 \lambda_{0} \lambda_{2}\right)+16 \alpha^{2} \lambda_{2}^{3}\left(\lambda_{1}^{2}+13 \lambda_{0} \lambda_{2}\right)+\alpha^{8} \lambda_{0}^{3}\left(\lambda_{1}^{2}+14 \lambda_{0} \lambda_{2}\right) \\
& \left.+2 \alpha^{4} \lambda_{2}\left(\lambda_{1}^{4}+10 \lambda_{0} \lambda_{1}^{2} \lambda_{2}+88 \lambda_{0}^{2} \lambda_{2}^{2}\right)\right) \lambda_{3}^{2} s_{1} s_{3}^{4}+\alpha^{3} \lambda_{1}\left(\alpha^{4} \lambda_{0}^{2}+4 \lambda_{2}^{2}+\alpha^{2}\left(\lambda_{1}^{2}+4 \lambda_{0} \lambda_{2}\right)\right) \\
& \times\left(128 \lambda_{2}^{7}-96 \alpha^{2} \lambda_{2}^{5}\left(\lambda_{1}^{2}-4 \lambda_{0} \lambda_{2}\right)+\alpha^{12} \lambda_{0}^{5}\left(2 \lambda_{0} \lambda_{2}-\lambda_{1}^{2}\right)+\alpha^{10} \lambda_{0}^{3}\left(24 \lambda_{0}^{2} \lambda_{2}^{2}-\lambda_{1}^{4}\right.\right. \\
& \left.-14 \lambda_{0} \lambda_{1}^{2} \lambda_{2}\right)+2 \alpha^{8} \lambda_{2}\left(60 \lambda_{0}^{2} \lambda_{2}^{2}-5 \lambda_{1}^{4}-36 \lambda_{0} \lambda_{1}^{2} \lambda_{2}\right)+8 \alpha^{4} \lambda_{2}^{3}\left(60 \lambda_{0}^{2} \lambda_{2}^{2}-3 \lambda_{1}^{4}\right. \\
& \left.\left.-26 \lambda_{0} \lambda_{1}^{2} \lambda_{2}\right)-2 \alpha^{6} \lambda_{2}\left(\lambda_{1}^{6}+14 \lambda_{0} \lambda_{1}^{4} \lambda_{2}+88 \lambda_{0}^{2} \lambda_{1}^{2} \lambda_{2}^{2}-160 \lambda_{0}^{3} \lambda_{2}^{3}\right)\right) \lambda_{3}^{2} s_{3}^{5} \\
& \left.-\alpha^{5} \lambda_{1}\left(\alpha^{2} \lambda_{0}+2 \lambda_{2}\right)^{4} \alpha^{4} \lambda_{0}^{2}+4 \lambda_{2}^{2}+\alpha^{2}\left(\lambda_{1}^{2}+4 \lambda_{0} \lambda_{2}\right)\right)^{2} \times\left(\lambda_{3}^{3} s_{1}^{2} s_{3}^{5}-2 \alpha^{6} \lambda_{1}^{2}\left(\alpha^{2} \lambda_{0}+2 \lambda_{2}\right)^{3}\right. \\
& \times\left(\alpha^{4} \lambda_{0}^{2}+4 \lambda_{2}^{2}+\alpha^{2}\left(\lambda_{1}^{2}+4 \lambda_{0} \lambda_{2}\right)\right)^{2} \lambda_{3}^{3} s_{1} s_{3}^{6}-\alpha^{7} \lambda_{1}^{3}\left(\alpha^{2} \lambda_{0}+2 \lambda_{2}\right)^{2}\left(\alpha^{4} \lambda_{0}^{2}+4 \lambda_{2}^{2}\right. \\
& \left.+\alpha^{2}\left(\lambda_{1}^{2}+4 \lambda_{0} \lambda_{2}\right)\right)^{2} \lambda_{3}^{3} s_{3}^{7}=0 \\
& -2 \lambda_{2}\left(\alpha^{2} \lambda_{0}+2 \lambda_{2}\right)^{3}\left(\alpha^{16} \lambda_{0}^{8}+256 \lambda_{2}^{8}+16 \alpha^{12} \lambda_{0}^{5} \lambda_{2}\left(\lambda_{1}^{2}+7 \lambda_{0} \lambda_{2}\right)+\alpha^{14} \lambda_{0}^{6}\left(\lambda_{1}^{2}+16 \lambda_{0} \lambda_{2}\right)\right. \\
& +64 \alpha^{2} \lambda_{2}^{6}\left(3 \lambda_{1}^{2}+16 \lambda_{0} \lambda_{2}\right)+32 \alpha^{4} \lambda_{2}^{4}\left(\lambda_{1}^{4}+16 \lambda_{0} \lambda_{1}^{2} \lambda_{2}+56 \lambda_{0}^{2} \lambda_{2}^{2}\right)+4 \alpha^{10} \lambda_{0}^{3} \lambda_{2}\left(\lambda_{1}^{4}\right. \\
& \left.+25 \lambda_{0} \lambda_{1}^{2} \lambda_{2}+112 \lambda_{0}^{2} \lambda_{2}^{2}\right)+8 \alpha^{8} \lambda_{0}^{2} \lambda_{2}^{2}\left(3 \lambda_{1}^{4}+40 \lambda_{0} \lambda_{1}^{2} \lambda_{2}+140 \lambda_{0}^{2} \lambda_{2}^{2}\right)+4 \alpha^{6} \lambda_{2}^{2}\left(\lambda_{1}^{6}\right. \\
& \left.\left.+12 \lambda_{0} \lambda_{1}^{4} \lambda_{2}+140 \lambda_{0}^{2} \lambda_{1}^{2} \lambda_{2}^{2}+448 \lambda_{0}^{3} \lambda_{2}^{3}\right)\right) r_{1}-2 \lambda_{1} \lambda_{2}\left(\alpha^{2} \lambda_{0}+2 \lambda_{2}\right)^{2}\left(\alpha^{16} \lambda_{0}^{8}+256 \lambda_{2}^{8}\right. \\
& +16 \alpha^{12} \lambda_{0}^{5} \lambda_{2}\left(\lambda_{1}^{2}+7 \lambda_{0} \lambda_{2}\right)+\alpha^{14} \lambda_{0}^{6}\left(\lambda_{1}^{2}+16 \lambda_{0} \lambda_{2}\right)+64 \alpha^{2} \lambda_{2}^{6}\left(3 \lambda_{1}^{2}+16 \lambda_{0} \lambda_{2}\right) \\
& +32 \alpha^{4} \lambda_{2}^{4}\left(\lambda_{1}^{4}+16 \lambda_{0} \lambda_{1}^{2} \lambda_{2}+56 \lambda_{0}^{2} \lambda_{2}^{2}\right)+4 \alpha^{10} \lambda_{0}^{3} \lambda_{2}\left(\lambda_{1}^{4}+25 \lambda_{0} \lambda_{1}^{2} \lambda_{2}+112 \lambda_{0}^{2} \lambda_{2}^{2}\right) \\
& +8 \alpha^{8} \lambda_{0}^{2} \lambda_{2}^{2}\left(3 \lambda_{1}^{4}+40 \lambda_{0} \lambda_{1}^{2} \lambda_{2}+140 \lambda_{0}^{2} \lambda_{2}^{2}\right)+4 \alpha^{6} \lambda_{2}^{2}\left(\lambda_{1}^{6}+12 \lambda_{0} \lambda_{1}^{4} \lambda_{2}+140 \lambda_{0}^{2} \lambda_{1}^{2} \lambda_{2}^{2}\right.
\end{aligned}
$$




$$
\begin{aligned}
& \left.\left.+448 \lambda_{0}^{3} \lambda_{2}^{3}\right)\right) s_{1}+2 \alpha \lambda_{2}\left(\alpha^{2} \lambda_{0}+2 \lambda_{2}\right)\left(512 \lambda_{0} \lambda_{2}^{9}+\alpha^{16} \lambda_{0}^{8}\left(2 \lambda_{0} \lambda_{2}-\lambda_{1}^{2}\right)-128 \alpha^{2} \lambda_{2}^{6}\left(\lambda_{1}^{4}\right.\right. \\
& \left.+\lambda_{0} \lambda_{1}^{2} \lambda_{2}-16 \lambda_{0}^{2} \lambda_{2}^{2}\right)+\alpha^{14} \lambda_{0}^{6}\left(32 \lambda_{0}^{2} \lambda_{2}^{2}-\lambda_{1}^{4}-16 \lambda_{0} \lambda_{1}^{2} \lambda_{2}\right)+2 \alpha^{12} \lambda_{0}^{5} \lambda_{2}\left(112 \lambda_{0}^{2} \lambda_{2}^{2}\right. \\
& \left.-9 \lambda_{1}^{4}-52 \lambda_{0} \lambda_{1}^{2} \lambda_{2}\right)-32 \alpha^{4} \lambda_{2}^{4}\left(\lambda_{1}^{6}+13 \lambda_{0} \lambda_{1}^{4} \lambda_{2}+16 \lambda_{0}^{2} \lambda_{1}^{2} \lambda_{2}^{2}-112 \lambda_{0}^{3} \lambda_{2}^{3}\right) \\
& +4 \alpha^{10} \lambda_{0}^{3} \lambda_{2}\left(224 \lambda_{0}^{3} \lambda_{2}^{3}-90 \lambda_{0}^{2} \lambda_{1}^{2} \lambda_{2}^{2}-\lambda_{1}^{6}-28 \lambda_{0} \lambda_{1}^{4} \lambda_{2}\right)+4 \alpha^{8} \lambda_{0}^{2} \lambda_{2}^{2}\left(560 \lambda_{0}^{3} \lambda_{2}^{3}-7 \lambda_{1}^{6}\right. \\
& \left.-84 \lambda_{0} \lambda_{1}^{4} \lambda_{2}-180 \lambda_{0}^{2} \lambda_{1}^{2} \lambda_{2}^{2}\right)-4 \alpha^{6} \lambda_{2}^{2}\left(\lambda_{1}^{8}+14 \lambda_{0} \lambda_{1}^{6} \lambda_{2}+132 \lambda_{0}^{2} \times \lambda_{1}^{4} \lambda_{2}^{2}+208 \lambda_{0}^{3} \lambda_{1}^{2} \lambda_{2}^{3}\right. \\
& \left.\left.-896 \lambda_{0}^{4} \lambda_{2}^{4}\right)\right) s_{3}-\alpha\left(\alpha^{2} \lambda_{0}+2 \lambda_{2}\right)^{3}\left(\alpha^{16} \lambda_{0}^{8}+256 \lambda_{2}^{8}+16 \alpha^{12} \lambda_{0}^{5} \lambda_{2}\left(\lambda_{1}^{2}+7 \lambda_{0} \lambda_{2}\right)\right. \\
& +\alpha^{14} \lambda_{0}^{6}\left(\lambda_{1}^{2}+16 \lambda_{0} \lambda_{2}\right)+64 \alpha^{2} \lambda_{2}^{6}\left(3 \lambda_{1}^{2}+16 \lambda_{0} \lambda_{2}\right)+32 \alpha^{4} \lambda_{2}^{4}\left(\lambda_{1}^{4}+16 \lambda_{0} \lambda_{1}^{2} \lambda_{2}+56 \lambda_{0}^{2} \lambda_{2}^{2}\right) \\
& +4 \alpha^{10} \lambda_{0}^{3} \lambda_{2}\left(\lambda_{1}^{4}+25 \lambda_{0} \times \lambda_{1}^{2} \lambda_{2}+112 \lambda_{0}^{2} \lambda_{2}^{2}\right)+8 \alpha^{8} \lambda_{0}^{2} \lambda_{2}^{2}\left(3 \lambda_{1}^{4}+40 \lambda_{0} \lambda_{1}^{2} \lambda_{2}+140 \lambda_{0}^{2} \lambda_{2}^{2}\right) \\
& \left.+4 \alpha^{6} \lambda_{2}^{2}\left(\lambda_{1}^{6}+12 \lambda_{0} \lambda_{1}^{4} \lambda_{2}+140 \lambda_{0}^{2} \lambda_{1}^{2} \lambda_{2}^{2}+448 \lambda_{0}^{3} \lambda_{2}^{3}\right)\right) \lambda_{3} s_{1}^{2} s_{3}-2 \alpha^{4} \lambda_{1}\left(\alpha^{2} \lambda_{0}+2 \lambda_{2}\right)^{2} \\
& \times\left(\alpha^{14} \lambda_{0}^{8}+128 \lambda_{2}^{6}\left(\lambda_{1}^{2}+2 \lambda_{0} \lambda_{2}\right)+\alpha^{12} \lambda_{0}^{6}\left(\lambda_{1}^{2}+16 \lambda_{0} \lambda_{2}\right)+4 \alpha^{8} \lambda_{0}^{3} \lambda_{2}\left(\lambda_{1}^{2}+5 \lambda_{0} \lambda_{2}\right)\right. \\
& \times\left(\lambda_{1}^{2}+20 \lambda_{0} \lambda_{2}\right)+4 \alpha^{10} \lambda_{0}^{5} \lambda_{2}\left(4 \lambda_{1}^{2}+27 \lambda_{0} \lambda_{2}\right)+32 \alpha^{2} \lambda_{2}^{4}\left(\lambda_{1}^{4}+13 \lambda_{0} \lambda_{1}^{2} \lambda_{2}+26 \lambda_{0}^{2} \lambda_{2}^{2}\right) \\
& +4 \alpha^{6} \lambda_{0}^{2} \lambda_{2}^{2}\left(7 \lambda_{1}^{4}+78 \lambda_{0} \lambda_{1}^{2} \lambda_{2}+220 \lambda_{0}^{2} \lambda_{2}^{2}\right)+4 \alpha^{4} \lambda_{2}^{2}\left(\lambda_{1}^{6}+14 \lambda_{0} \lambda_{1}^{4} \lambda_{2}+128 \lambda_{0}^{2} \lambda_{1}^{2} \lambda_{2}^{2}\right. \\
& \left.\left.+288 \lambda_{0}^{3} \lambda_{2}^{3}\right)\right) \lambda_{3} s_{1} s_{3}^{2}+\alpha\left(512 \alpha^{2} \lambda_{2}^{9}\left(\lambda_{1}^{2}-16 \lambda_{0} \lambda_{2}\right)-2048 \lambda_{2}^{1} 1+\alpha^{20} \lambda_{0}^{9}\left(2 \lambda_{0} \lambda_{2}-\lambda_{1}^{2}\right)\right. \\
& +\alpha^{18} \lambda_{0}^{7}\left(2 \lambda_{0} \lambda_{2}-\lambda_{1}^{2}\right)\left(\lambda_{1}^{2}+16 \lambda_{0} \lambda_{2}\right)+256 \alpha^{4} \lambda_{2}^{7}\left(\lambda_{1}^{4}+9 \lambda_{0} \lambda_{1}^{2} \lambda_{2}-54 \lambda_{0}^{2} \lambda_{2}^{2}\right) \\
& +8 \alpha^{16} \lambda_{0}^{6} \lambda_{2}\left(27 \lambda_{0}^{2} \lambda_{2}^{2}-2 \lambda_{1}^{4}-9 \lambda_{0} \lambda_{1}^{2} \lambda_{2}\right)+32 \alpha^{6} \lambda_{2}^{5}\left(\lambda_{1}^{6}+14 \lambda_{0} \lambda_{1}^{4} \lambda_{2}+128 \lambda_{0}^{2} \lambda_{1}^{2} \lambda_{2}^{2}\right. \\
& \left.-384 \lambda_{0}^{3} \lambda_{2}^{3}\right)+16 \alpha^{12} \lambda_{0}^{3} \lambda_{2}^{2} \times\left(84 \lambda_{0}^{3} \lambda_{2}^{3}-2 \lambda_{1}^{6}-15 \lambda_{0} \lambda_{1}^{4} \lambda_{2}+16 \lambda_{0}^{2} \lambda_{1}^{2} \lambda_{2}^{2}\right)-32 \alpha^{8} \lambda_{0} \lambda_{2}^{4}\left(\lambda_{1}^{6}\right. \\
& \left.-4 \lambda_{0} \lambda_{1}^{4} \lambda_{2}-116 \lambda_{0}^{2} \lambda_{1}^{2} \lambda_{2}^{2}+168 \lambda_{0}^{3} \lambda_{2}^{3}\right)+4 \alpha^{14} \lambda_{0}^{4} \lambda_{2}\left(192 \lambda_{0}^{3} \lambda_{2}^{3}-\lambda_{1}^{6}-23 \lambda_{0} \lambda_{1}^{4} \lambda_{2}\right. \\
& \left.\left.-32 \lambda_{0}^{2} \lambda_{1}^{2} \lambda_{2}^{2}\right)+4 \alpha^{10} \lambda_{0} \lambda_{1}^{2} \lambda_{2}^{2}\left(432 \lambda_{0}^{3} \lambda_{2}^{3}-\lambda_{1}^{6}-18 \lambda_{0} \lambda_{1}^{4} \lambda_{2}-60 \lambda_{0}^{2} \lambda_{1}^{2} \lambda_{2}^{2}\right)\right) \lambda_{3} s_{3}^{3} \\
& +2 \alpha^{3} \lambda_{2}\left(\alpha^{2} \lambda_{0}+2 \lambda_{2}\right)^{4}\left(\alpha^{4} \lambda_{0}^{2}+4 \lambda_{2}^{2}+\alpha^{2}\left(\lambda_{1}^{2}+4 \lambda_{0} \lambda_{2}\right)\right)\left(\alpha^{6} \lambda_{0}^{3}+12 \alpha^{2} \lambda_{0} \lambda_{2}^{2}+8 \lambda_{2}^{3}\right. \\
& \left.+\alpha^{4} \lambda_{0}\left(6 \lambda_{0} \lambda_{2}-\lambda_{1}^{2}\right)\right) \lambda_{3}^{2} s_{1}^{2} s_{3}^{3}+8 \alpha^{4} \lambda_{1} \lambda_{2}\left(\alpha^{2} \lambda_{0}+2 \lambda_{2}\right)\left(\alpha^{4} \lambda_{0}^{2}+4 \lambda_{2}^{2}+\alpha^{2}\left(\lambda_{1}^{2}+4 \lambda_{0} \lambda_{2}\right)\right) \\
& \times\left(\alpha^{10} \lambda_{0}^{5}+10 \alpha^{8} \lambda_{0}^{4} \lambda_{2}+32 \lambda_{2}^{5}+8 \alpha^{2} \lambda_{2}^{3}\left(\lambda_{1}^{2}+10 \lambda_{0} \lambda_{2}\right)+2 \alpha^{6} \lambda_{0}^{2} \lambda_{2}\left(\lambda_{1}^{2}+20 \lambda_{0} \lambda_{2}\right)\right. \\
& \left.+\alpha^{4} \lambda_{2}\left(\lambda_{1}^{4}+8 \lambda_{0} \lambda_{1}^{2} \lambda_{2}+80 \lambda_{0}^{2} \lambda_{2}^{2}\right)\right) \lambda_{3}^{2} s_{1} s_{3}^{4}-2 \alpha^{3} \lambda_{2}\left(\alpha^{4} \lambda_{0}^{2}+4 \lambda_{2}^{2}+\alpha^{2}\left(\lambda_{1}^{2}+4 \lambda_{0} \lambda_{2}\right)\right) \\
& \times\left(\alpha^{14} \lambda_{0}^{7}+128 \lambda_{2}^{7}-32 \alpha^{2} \lambda_{2}^{5}\left(\lambda_{1}^{2}-14 \lambda_{0} \lambda_{2}\right)+2 \alpha^{12} \lambda_{0}^{5}\left(7 \lambda_{0} \lambda_{2}-\lambda_{1}^{2}\right)-24 \alpha^{4} \lambda_{2}^{3}\left(\lambda_{1}^{4}\right.\right. \\
& \left.+4 \lambda_{0} \lambda_{1}^{2} \lambda_{2}-28 \lambda_{0}^{2} \lambda_{2}^{2}\right)+\alpha^{10} \lambda_{0}^{3}\left(84 \lambda_{0}^{2} \lambda_{2}^{2}-\lambda_{1}^{4}-18 \lambda_{0} \lambda_{1}^{2} \lambda_{2}\right)+2 \alpha^{8} \lambda_{0}^{2} \lambda_{2}\left(140 \lambda_{0}^{2} \lambda_{2}^{2}-5 \lambda_{1}^{4}\right. \\
& \left.\left.-32 \lambda_{0} \lambda_{1}^{2} \lambda_{2}\right)-4 \alpha^{6} \lambda_{2}\left(\lambda_{1}^{6}+7 \lambda_{0} \lambda_{1}^{4} \lambda_{2}+28 \lambda_{0}^{2} \lambda_{1}^{2} \lambda_{2}^{2}-140 \lambda_{0}^{3} \lambda_{2}^{3}\right)\right) \lambda_{3}^{2} s_{3}^{5}+\alpha^{5}\left(\alpha^{2} \lambda_{0}+2 \lambda_{2}\right)^{2} \\
& \times\left(\alpha^{4} \lambda_{0}^{2}+4 \lambda_{2}^{2}+\alpha^{2}\left(\lambda_{1}^{2}+4 \lambda_{0} \lambda_{2}\right)\right)^{2}\left(\alpha^{6} \lambda_{0}^{3}+6 \alpha^{4} \lambda_{0}^{2} \lambda_{2}+8 \lambda_{2}^{3}\right. \\
& \left.+2 \alpha^{2} \lambda_{2}\left(\lambda_{1}^{2}+6 \lambda_{0} \lambda_{2}\right)\right) \lambda_{3}^{3} s_{1}^{2} s_{3}^{5}+2 \alpha^{6} \lambda_{1}\left(\alpha^{2} \lambda_{0}+2 \lambda_{2}\right)\left(\alpha^{4} \lambda_{0}^{2}+4 \lambda_{2}^{2}+\alpha^{2}\left(\lambda_{1}^{2}+4 \lambda_{0} \lambda_{2}\right)\right)^{2} \\
& \times\left(\alpha^{6} \lambda_{0}^{3}+6 \alpha^{4} \lambda_{0}^{2} \lambda_{2}+8 \lambda_{2}^{3}+2 \alpha^{2} \lambda_{2}\left(\lambda_{1}^{2}+6 \lambda_{0} \lambda_{2}\right)\right) \lambda_{3}^{3} s_{1} s_{3}^{6}+\alpha^{7} \lambda_{1}^{2}\left(\alpha^{4} \lambda_{0}^{2}+4 \lambda_{2}^{2}\right. \\
& \left.+\alpha^{2}\left(\lambda_{1}^{2}+4 \lambda_{0} \lambda_{2}\right)\right)^{2}\left(\alpha^{6} \lambda_{0}^{3}+6 \alpha^{4} \lambda_{0}^{2} \lambda_{2}+8 \lambda_{2}^{3}+2 \alpha^{2} \lambda_{2}\left(\lambda_{1}^{2}+6 \lambda_{0} \lambda_{2}\right)\right) \lambda_{3}^{3} s_{3}^{7}=0 .
\end{aligned}
$$

\section{Appendix}

The stationary solutions of the system (16):

$$
\begin{aligned}
& \left\{s_{1}=-\frac{\sqrt{2 \alpha^{2} \lambda_{0}^{2}+\lambda_{1}^{2}-\lambda_{1} \sqrt{4 \alpha^{2} \lambda_{0}^{2}+\lambda_{1}^{2}}}\left(\lambda_{1}+\sqrt{4 \alpha^{2} \lambda_{0}^{2}+\lambda_{1}^{2}}\right) \sqrt{\lambda_{0}-\lambda_{3} s_{2}^{2}}}{2 \sqrt{2} \alpha^{2} \lambda_{0}^{2} \sqrt{\lambda_{3}}},\right. \\
& s_{3}=0, \quad r_{1}=-\frac{\sqrt{2 \alpha^{2} \lambda_{0}^{2}+\lambda_{1}^{2}-\lambda_{1} \sqrt{4 \alpha^{2} \lambda_{0}^{2}+\lambda_{1}^{2}}} \sqrt{\lambda_{0}-\lambda_{3} s_{2}^{2}}}{2 \sqrt{2} \alpha^{2} \lambda_{0} \sqrt{\lambda_{3}}}, \\
& \left.r_{2}=-\frac{\left(\lambda_{1}-\sqrt{4 \alpha^{2} \lambda_{0}^{2}+\lambda_{1}^{2}}\right) s_{2}}{2 \alpha^{2} \lambda_{0}}, \quad r_{3}=0\right\},
\end{aligned}
$$




$$
\begin{aligned}
& \left\{s_{1}=\frac{\sqrt{2 \alpha^{2} \lambda_{0}^{2}+\lambda_{1}^{2}-\lambda_{1} \sqrt{4 \alpha^{2} \lambda_{0}^{2}+\lambda_{1}^{2}}}\left(\lambda_{1}+\sqrt{4 \alpha^{2} \lambda_{0}^{2}+\lambda_{1}^{2}}\right) \sqrt{\lambda_{0}-\lambda_{3} s_{2}^{2}}}{2 \sqrt{2} \alpha^{2} \lambda_{0}^{2} \sqrt{\lambda_{3}}},\right. \\
& s_{3}=0, \quad r_{1}=\frac{\sqrt{2 \alpha^{2} \lambda_{0}^{2}+\lambda_{1}^{2}-\lambda_{1} \sqrt{4 \alpha^{2} \lambda_{0}^{2}+\lambda_{1}^{2}}} \sqrt{\lambda_{0}-\lambda_{3} s_{2}^{2}}}{2 \sqrt{2} \alpha^{2} \lambda_{0} \sqrt{\lambda_{3}}}, \\
& \left.\left.r_{2}=-\frac{\left(\lambda_{1}-\sqrt{4 \alpha^{2} \lambda_{0}^{2}+\lambda_{1}^{2}}\right) s_{2}}{2 \alpha^{2} \lambda_{0}}, \quad r_{3}=0\right\}\right\} .
\end{aligned}
$$

Here $\lambda_{2}=-\left(2 \alpha^{2} \lambda_{0}^{2}+\lambda_{1}^{2}+\lambda_{1} \sqrt{4 \alpha^{2} \lambda_{0}^{2}+\lambda_{1}^{2}}\right) /\left(4 \lambda_{0}\right)$.

$$
\begin{aligned}
& \left\{s_{1}=-\frac{\sqrt{2 \alpha^{2} \lambda_{0}^{2}+\lambda_{1}^{2}+\lambda_{1} \sqrt{4 \alpha^{2} \lambda_{0}^{2}+\lambda_{1}^{2}}}\left(\lambda_{1}-\sqrt{4 \alpha^{2} \lambda_{0}^{2}+\lambda_{1}^{2}}\right) \sqrt{\lambda_{0}-\lambda_{3} s_{2}^{2}}}{2 \sqrt{2} \alpha^{2} \lambda_{0}^{2} \sqrt{\lambda_{3}}},\right. \\
& s_{3}=0, \quad r_{1}=-\frac{\sqrt{2 \alpha^{2} \lambda_{0}^{2}+\lambda_{1}^{2}+\lambda_{1} \sqrt{4 \alpha^{2} \lambda_{0}^{2}+\lambda_{1}^{2}}} \sqrt{\lambda_{0}-\lambda_{3} s_{2}^{2}}}{2 \sqrt{2} \alpha^{2} \lambda_{0} \sqrt{\lambda_{3}}}, \\
& \left.r_{2}=-\frac{\left(\lambda_{1}+\sqrt{4 \alpha^{2} \lambda_{0}^{2}+\lambda_{1}^{2}}\right) s_{2}}{2 \alpha^{2} \lambda_{0}}, r_{3}=0\right\}, \\
& \left\{s_{1}=\frac{\sqrt{2 \alpha^{2} \lambda_{0}^{2}+\lambda_{1}^{2}+\lambda_{1} \sqrt{4 \alpha^{2} \lambda_{0}^{2}+\lambda_{1}^{2}}}\left(\lambda_{1}-\sqrt{4 \alpha^{2} \lambda_{0}^{2}+\lambda_{1}^{2}}\right) \sqrt{\lambda_{0}-\lambda_{3} s_{2}^{2}}}{2 \sqrt{2} \alpha^{2} \lambda_{0}^{2} \sqrt{\lambda_{3}}},\right. \\
& s_{3}=0, \quad r_{1}=\frac{\sqrt{2 \alpha^{2} \lambda_{0}^{2}+\lambda_{1}^{2}+\lambda_{1} \sqrt{4 \alpha^{2} \lambda_{0}^{2}+\lambda_{1}^{2}}} \sqrt{\lambda_{0}-\lambda_{3} s_{2}^{2}}}{2 \alpha^{2} \lambda_{0} \sqrt{\lambda_{3}}}, \\
& \left.\left.r_{2}=-\frac{\left(\lambda_{1}+\sqrt{4 \alpha^{2} \lambda_{0}^{2}+\lambda_{1}^{2}}\right) s_{2}}{2 \alpha^{2} \lambda_{0}}, r_{3}=0\right\}\right\} .
\end{aligned}
$$

Here $\lambda_{2}=-\left(2 \alpha^{2} \lambda_{0}^{2}+\lambda_{1}^{2}-\lambda_{1} \sqrt{4 \alpha^{2} \lambda_{0}^{2}+\lambda_{1}^{2}}\right) /\left(4 \lambda_{0}\right)$.

[1] Borisov A.V., Mamaev I.S., Sokolov V.V., A new integrable case on so(4), Dokl. Akad. Nauk, 2001, V.381, N 5, 614-615 (English transl.: Dokl. Phys., 2001, V.46, N 12, 888-889).

[2] Cox D., Little J., O'Shea D., Ideals, varieties, and algorithms, New York, Springer-Verlag, 1997.

[3] Harlamov P.V., On motion in a fluid of a body bounded by a multiply connected surface, Prikl. Mekh. Tekhn. Fiz., 1963, N 4, 17-29 (in Russian).

[4] Kirchhoff G., Vorlesungen über Mathematische Physik. Mechanik, Leipzig, B. Teubner, Bd. 1, 1897.

[5] Kovalev Yu.M., On the stability of steady helical motions in a fluid of a body bounded by a multiply connected surface, J. Appl. Math. Mech., 1968, V.32, N 2, 272-275.

[6] Kozlov V.V., Onishchenko D.A., The motion in a perfect fluid of a body containing a moving point mass, J. Appl. Math. Mech., 2003, V.67, N 4, 553-564.

[7] Lamb H., Hydrodinamics, New York, Dover Publ., 1945.

[8] Lyapunov A.M., On permanent helical motions of a rigid body in fluid, Moscow-Leningrad, USSR Acad. Sci., Collected Works, Vol. 1, 1954 (in Russian).

[9] Lyapunov A.M., Stability of motion, New York, Academic Press, 1966.

[10] Oparina E.I., Troshkin O.V., Stability of Kolmogorov flow in a channel with rigid walls, Dokl. Akad. Nauk, 2004, V.398, N 4, 487-491 (English transl.: Dokl. Phys., 2004, V.49, N 10, 583-587).

[11] Sokolov V.V., A new integrable case for the Kirchhoff equation, Teoret. Mat. Fiz., 2001, V.129, N 1, 31-37 (English transl.: Theoret. and Math. Phys., 2001, V.129, N 1, 1335-1340).

[12] Rumyantsev V.V., A comparison of three methods of constructing Lyapunov functions, J. Appl. Math. Mech., 1995, V.59, N 6, 873-877.

[13] Ryabov P.E., Bifurcations of first integrals in the Sokolov case, Teoret. Mat. Fiz., 2003, V.134, N 2, 207-226 (English transl.: Theoret. and Math. Phys., 2003, V.34, N 2, 181-197). 